Dominik Berrens*

\title{
The Old Man and the Bee - Zur Entwicklung eines literarischen Motivs
}

https://doi.org/10.1515/phil-2020-0104

\begin{abstract}
Although bees are a frequent motif in ancient literature, the people who work with bees are often left in the background. An exception is the motif of the older man on his - usually small - farm who lives from and with his bees. The article shows that this motif is a topos that appears in various texts of Greek and Latin literature of the imperial period. Depending on the intention behind these representations, different elements of the motif may be emphasised or omitted. These variations, and how the motif develops, are here shown through the example of different passages.
\end{abstract}

Keywords: Greis, Biene, Kaiserzeitliche Literatur, Topos

\section{Einführung}

In der antiken Literatur allgemein, keineswegs nur in den naturkundlichen und agronomischen Schriften, spielen Bienen eine wichtige Rolle. Weniger häufig werden dagegen die Menschen thematisiert, die sich direkt mit ihnen befassen.

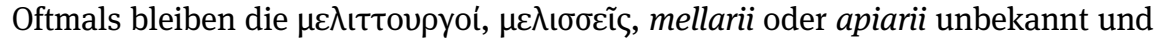
weitgehend unspezifisch. ${ }^{1}$ Dies ist vermutlich auf die Tatsache zurückzuführen, dass in den meisten - und speziell vor allem den agronomischen Texten - darunter bestimmte Sklaven zu verstehen sind, die sich um die Bienenstöcke auf den

1 Diese Begriffe bezeichnen im weitesten Sinne „Imker“ bzw. „Bienenzüchter“, d.h. Menschen, die Honig von kultivierten Bienenstöcken gewinnen. Das Honigsammeln von wildlebenden Bienen, das sogenannten Zeideln, wird in antiken Texten ebenfalls erwähnt (vgl. z.B. Davies/Kathiritamby 1986, 50-52; Crane 1999, 45-46). Bekannt ist vor allem ein Gleichnis, das in verschiedenen Epen in ähnlicher Form enthalten ist (Hom. Il. 12,167-172; Apoll. Rhod. 2,130-136; Verg. Aen. 12,586-592; Stat. Theb. 10,574-579; vgl. auch Reitz 1996, 46-50; Berrens 2018, 255-258).

*Kontaktperson: Dominik Berrens, Leopold-Franzens-Universität Innsbruck, Langer Weg 11, A-6020 Innsbruck, E-Mail: dominik.berrens@uibk.ac.at

Ә Open Access. (C) 2020 Dominik Berrens, publiziert von De Gruyter. (c))BY Dieses Werk ist lizenziert unter der Creative Commons Attribution 4.0 Lizenz. 
Latifundien zu kümmern hatten. ${ }^{2}$ In den wenigen Texten, in denen ein Imker als etwas deutlicher umrissene Figur auftritt, lassen sich allerdings interessante Parallelen und topische Eigenschaften erkennen, die dem Imker als literarischer Figur zugeschrieben werden. Vor allem in der griechischen und lateinischen Literatur der Kaiserzeit begegnet uns der Imker als meist älterer Mann, der von und mit seinen Bienen auf einem kleinen Landgut lebt. Je nach Intention und Gattung des jeweiligen Textes können dabei ganz unterschiedliche Aspekte und zusätzliche Eigenschaften hervorgehoben werden. Im Folgenden soll gezeigt werden, dass es sich bei der Figur des alten Imkers um ein topisches Motiv handelt. Darüber hinaus sollen die Einzelzüge des Motivs sowie ihre Entwicklung durch die Interpretation verschiedener Belegstellen in grob chronologischer Reihenfolge herausgearbeitet werden.

\section{Vorläufer des Motivs}

Das erste Mal in der erhaltenen antiken Literatur taucht das Motiv eines Bienenzüchters in Ansätzen in den Wolken des Aristophanes auf. ${ }^{3}$ Gleich zu Beginn wird uns der Protagonist Strepsiades gezeigt, den seine finanziellen Sorgen, die durch das verschwenderische Leben seines Sohnes und seiner Frau entstanden sind, nicht schlafen lassen. Wehmütig sehnt er sich in diesem Zusammenhang in sein altes Leben auf dem Lande zurück, wo ihm verschiedene agronomische Aktivitäten, darunter die erstgenannte Bienenzucht, ein weitgehend sorgenfreies Auskommen ermöglichten (Nub. 41-52):

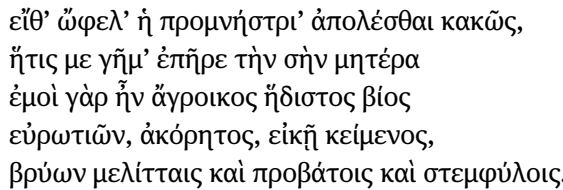

2 Tatsächlich ist über diese Berufsgruppe wenig bekannt. Columella, der sich auch zu den körperlichen und geistigen Eigenschaften der Sklaven für bestimmte Tätigkeiten in der Landwirtschaft äußert (Res rustica 1,9), bespricht den Imker nicht eigens. Es finden sich freilich Aussagen wie die, dass die Imker reinlich sein und zeitweise nicht sexuell aktiv sein dürfen (z.B. Verg. georg. 4,228230; Colum. 9,5,2; 9,14,3; Pall. agric. 1,37,4; 4,15,4; 5,7,5). Vgl. z.B. Olck (1897) 454-455; Sünskes Thompson (1997) 651; Berrens (2018) 61. Zur antiken Bienenzucht allgemein (vor allem auch zu archäologischen Belegen) vgl. z.B. Olck (1897); Fraser (1951); Jones (1976); Crane (1983) v.a. 45-56; Sünskes Thompson (1997); Crane (1999); Hollander (2019) 68-71 sowie Klek/Armbruster (19191926) zu einzelnen Autoren.

3 Smith (2014) 33 hält die Stelle für „one of the earliest literary representations of rustic Athenians". 


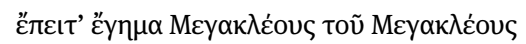

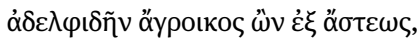

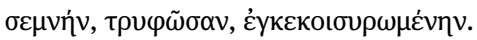

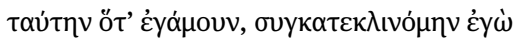

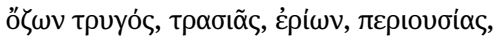

50

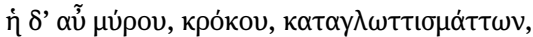

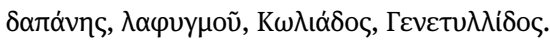

Wenn doch nur die Ehestifterin übel zugrunde ginge, die mich dazu überredet hat, deine ${ }^{4}$ Mutter zu heiraten. Denn ich hatte das allersüßeste Bauernleben, ungewaschen, unrasiert, ohne Ziel auf der faulen Haut liegend, überreich an Bienen, Schafen und ausgepressten Oliven. Dann habe ich die Nichte des Megakles, des Sohnes von Megakles geheiratet, obwohl ich vom Land kam, eine Frau aus der Stadt, vornehm, verschwenderisch und luxuriös wie Koisyra. Als ich sie heiratete, schlief ich mit ihr und roch nach Weinhefe, getrockneten Feigen, Wolle und Überfluss, sie dagegen nach Salbe, Safran, Zungenküssen, Verschwendung, Schlemmerei, Kolias und Genetyllis.

In dieser kurzen Passage entwirft Strepsiades einen Gegensatz zwischen dem idealisierten Landleben und dem als schlecht wahrgenommenen Leben in der Stadt. Besonders betont wird die Sparsamkeit und die Einfachheit des Land-

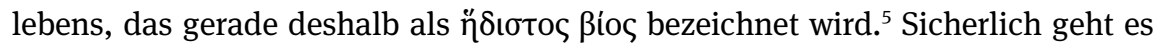
Aristophanes an dieser Stelle nicht darum, eine korrekte Darstellung eines Bauern und Imkers zu geben, sondern hier einen möglichst krassen Gegensatz zwischen dem von Strepsiades als positiv dargestellten Landleben und dem Leben seiner Frau zu zeigen. Das Motiv des alten Mannes, der mit seinen Bienen in Einklang lebt, ist hier auch eher nur in Ansätzen zu erkennen. Schließlich muss man davon ausgehen, dass Strepsiades, obgleich in der Gegenwart der Komödie schon älter, damals ja noch jünger war. Es stehen nicht die Bienen im Vordergrund der Schilderung, sondern sie sind ein gleichberechtigtes Element neben Schafen und Oliven sowie den später genannten Feigen (50), Wein (50) und Ziegen (71). ${ }^{6}$

Einen ähnlichen Katalog an landwirtschaftlichen Tätigkeiten, wie wir ihn bei Aristophanes finden, lässt Cicero den älteren Cato in seiner nach ihm benannten Schrift über das Alter aufzählen (Cato 54):

Nec vero segetibus solum et pratis et vineis et arbustis res rusticae laetae sunt, sed hortis etiam et pomariis tum pecudum pastu apium examinibus florum omnium varietate. Nec consitiones modo delectant sed etiam insitiones, quibus nihil invenit agri cultura sollertius.

4 Strepsiades spricht seinen schlafenden Sohn an.

5 Dies bedeutet aber wohl nicht, dass Strepsiades als arm zu gelten hat. Immerhin konnte er eine reiche Frau heiraten und hohe Kredite erhalten. Vgl. Dover (1968) xxvii-xxviii.

6 Vgl. z.B. Sommerstein (1982) 161. 


\begin{abstract}
Nicht nur wegen der Saatfelder, Wiesen, Weinberge und Baumpflanzungen ist die Landwirtschaft fruchtbar, sondern auch wegen der Gärten, Obstgärten und ganz besonders auch wegen der Viehweiden, der Bienenschwärme und der Vielfalt aller Blüten. Nicht nur Aussaaten bringen Freude, sondern auch Pfropfungen, die das Geschickteste sind, was die Landwirtschaft erfunden hat.
\end{abstract}

Die Bienenzucht wird hier wiederum nur als eine unter vielen Tätigkeiten genannt, auf die sich die Freude des älteren Landwirtes erstreckt, wenngleich sie immerhin zu der Gruppe gehört, die besondere Freude bringen können. Festzuhalten ist noch, dass die Bienenzucht in Catos eigener agronomischer Schrift De agricultura nicht behandelt wird.

Eine etwas spezifischere Darstellung von Bienenzüchtern enthält Varros Dia$\log$ Res rusticae. Das darin enthaltene Kapitel zur Bienenzucht ist gewissermaßen zweigeteilt. Zunächst erläutert Appius eher naturkundliche Aspekte der Bienenzucht, anschließend spricht Merula in einem umfangreicheren zweiten Teil praktischere Dinge an. Im Dialog selbst werden diese Teile als physica und de fructu bezeichnet $(3,16,9)$. Gleich zu Beginn des zweiten Teils berichtet Merula nun von erfolgreichen Bienenzüchtern. Dies sind zum einen Seius, der seine Bienenstöcke verpachtet und jedes Jahr je 5000 Pfund Honig gewonnen haben soll, ${ }^{7}$ sowie vor allem die Gebrüder Veianii (rust. 3,16,10-11):

... quem ${ }^{8}$ audivi dicentem duo milites se habuisse in Hispania fratres Veianios ex agro Falisco locupletis, quibus cum a patre relicta esset parva villa et agellus non sane maior iugero uno, hos circum villam totam alvaria fecisse et hortum habuisse ac relicum thymo et cytiso opsevisse et apiastro ... Hos numquam minus, ut peraeque ducerent, dena milia sestertia ex melle recipere esse solitos ...

... den ich sagen hörte, dass er zwei Soldaten, Brüder mit dem Namen Veianius, (in seiner Truppe) in Hispanien gehabt habe, die aus dem Faliskerland (stammten) und wohlhabend waren. Da diesen vom Vater nur ein kleines Landhaus und ein winziger Acker, wirklich nicht größer als ein einziger Morgen, zurückgelassen worden sei, hätten sie um das ganze Landhaus herum Bienenstöcke errichtet und einen Garten gehabt und den Rest mit Thymian, Schneckenklee und Melisse bepflanzt ... Diese sollen gewöhnlich niemals weniger als im Durchschnitt 10.000 Sesterzen aus dem Honig eingenommen haben ...

Die beiden Brüder Veianii scheinen zwar aus einfacheren Verhältnissen zu kommen, doch können sie durch ihr ökonomisches Geschick das Bestmögliche aus ihrem nur kleinen Landbesitz (agellus) herausholen. Dies gelingt ihnen, indem sie ein Produkt herstellen, das bei möglichst geringem Einsatz einen möglichst ho-

7 Laut Flach (2002) 273 entspricht dies 1637,25 kg.

8 Gemeint ist Varro, der sich selbst in den Dialogen auftreten lässt. Es spricht Merula. 
hen Ertrag verspricht, nämlich Honig. ${ }^{9}$ Dieses Ziel erreichen sie auch; immerhin werden sie als locupletes bezeichnet und ihnen wird ein durchschnittlicher Ertrag von 10.000 Sesterzen attestiert. ${ }^{10}$ Planvoll optimieren sie die Landnutzung, indem sie einen Garten anlegen und dann gezielt Pflanzen ansiedeln, die in der Antike als gute Produzenten für Honig galten. ${ }^{11}$ Die Brüder scheinen ansonsten keiner weiteren landwirtschaftlichen Betätigung nachgegangen zu sein und ihren Verkauf dahingegen optimiert zu haben, dass sie erst dann ihren Honig auf den Markt brachten, wenn die Preise ausreichend gut waren $(3,16,11),{ }^{12}$ eine Möglichkeit die Honig im Gegensatz zu vielen anderen schnell verderblichen landwirtschaftlichen Produkten bietet. Die Bienen werden in dieser Passage also in erster Linie als rentables Geschäftsmodell präsentiert und treten somit auch nicht als besonders detailliert beschriebene, eigenständige Akteure hervor. Es ist zudem festzuhalten, dass die Beschreibung eines Stück Landes, als zu nichts anderem mehr geeignet als zur Bienenzucht, nicht erst hier eingeführt wird. Im platonischen Dialog Kritias hält der namensgebende Sprecher einen längeren Monolog, in dem unter anderem der Naturraum Ur-Attikas mit dem Zustand in der Gegenwart des Dialogs verglichen wird. Dort heißt es, dass durch zahlreiche Überschwemmungen in den vergangenen 9000 Jahren der ursprünglich fruchtbare Boden weitgehend abgetragen worden sei. Eine Folge daraus sei eine weitgehende Entwaldung und schlechtere Böden für die Landwirtschaft. Die Bienenzucht wird dabei explizit als derjenige Bereich der Landwirtschaft genannt, der als ein-

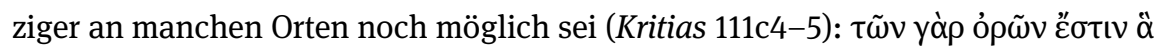

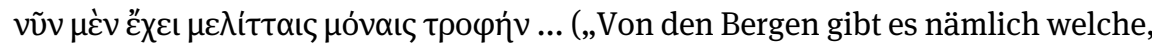
die jetzt nur noch Bienen Nahrung bieten ...“). Sicherlich ist hier vor allem an das Hymettos-Gebirge südöstlich von Athen gedacht, ${ }^{13}$ das in der gesamten Antike als Herkunft des besten Honigs galt. ${ }^{14}$ Ähnlich wie im Falle des ungünstigen Land-

9 Auch Plin. nat. 21,70 (res praecipui quaestus conpendiique) weist auf die lukrative Bienenzucht hin. Neben Honig gehört auch Wachs zu den Bienenprodukten, wenngleich dies weniger einträglich war (Cerae fructus quamvis aeris exigui non tamen omittendus; Colum. 9,16,1). Vgl. auch Hollander (2019) 69-70 für diese und weitere Stellen.

10 Dies scheint ein ordentliches Einkommen gewesen zu sein. Vgl. z.B. Klek/Armbruster (1920) 252; Guiraud (1997) 102; Hollander (2019) 69.

11 Thymian, Schneckenklee und Melisse finden sich in verschiedenen Listen von geeigneten Bienenweiden, z.B. in Aristot. hist. an. IX 40, 627 b 16-18; Varro rust. 3,16,13-14, Colum. 9,4; Plin. nat. 21,70-72; Pall. agric. 1,37,2-3. Thymian galt allgemein als die beste Pflanze für Honig: Z. B. Aristot. hist. an. IX 40, 627 a 1-2; Varro rust. 3,16,14; Verg. georg. 4,169; Colum. 9,4,2; Plin. nat. 11,38-39; dazu Theophr. h. plant. 6,2,3.

12 Vgl. Tilly (1973) 299; Flach (2002) 273.

13 Vgl. Nesselrath (2006) 202.

14 Z.B. Plin. nat. 11,32; Paus. 1,32,1; Geop. 15,7,1. 
gutes der Gebrüder Veianii wird hier also die Bienenzucht als letzte Möglichkeit genannt, überhaupt noch einen Gewinn aus dem Land zu ziehen, was sich jedoch als durchaus lukrativ und prestigeträchtig erweisen konnte.

\section{Vergil}

In den Werken Vergils finden sich verschiedene Darstellungen von Männern, die Bienen halten. Eine bekannte Figur ist die des Aristaeus, dessen Mythos im zweiten Teil (315-558) des vierten Buches der Georgica erzählt wird. Da es sich hierbei jedoch um eine mythische Figur handelt, die zumindest bei Vergil als junger Mann gezeigt wird und auch sonst wenig Ähnlichkeiten mit den übrigen hier behandelten Figuren hat, soll Aristaeus hier außen vor gelassen werden.

Eine für unser Motiv interessante Figur findet sich jedoch in der ersten Ekloge, in der Meliboeus auf den Freigelassenen Tityrus trifft. Während ersterer sein Land in Folge der Umverteilung nach dem Bürgerkrieg verlassen muss, darf Tityrus auf seinem Land bleiben. ${ }^{15}$ Als Meliboeus vom positiven Ausgang für Tityrus bei seiner Bitte um Freilassung vor Octavian erfahren hat (45), spricht er folgende Worte, in denen er Tityrus glücklich preist und sein Land als locus amoenus schildert $(1,46-58):^{16}$

Fortunate senex, ergo tua rura manebunt et tibi magna satis, quamvis lapis omnia nudus limosoque palus obducat pascua iunco: non insueta gravis temptabunt pabula fetas, nec mala vicini pecoris contagia laedent.

Fortunate senex, hic inter flumina nota et fontis sacros frigus captabis opacum; hinc tibi, quae semper, vicino ab limite saepes Hyblaeis apibus florem depasta salicti saepe levi somnum suadebit inire susurro; hinc alta sub rupe canet frondator ad auras, nec tamen interea raucae, tua cura, palumbes nec gemere aeria cessabit turtur ab ulmo.

Glücklicher Greis, also wird dir dein Landgut bleiben und es dir groß genug sein, obwohl alles nackter Stein ist und ein Sumpf mit schlammigen Binsen deine Weiden bedeckt: Un-

15 In der Ekloge wird vieles nur in Anspielung erzählt. Tityrus war offenbar ein Sklave, der nun durch Augustus das Land seines ehemaligen Herrn zugesprochen bekommen hat (1,40-45). Vgl. z.B. Coleman (1977) 80-82; Clausen (1994) 30-31.

16 Vgl. z.B. Putnam (1970) 46-51; Coleman (1977) 83; Thomas (1992) 53. 
gewohntes Futter wird deine trächtigen Tiere nicht locken und schlimme Ansteckungen des benachbarten Viehs werden ihnen nicht schaden. Glücklicher Greis, hier zwischen den bekannten Flüssen und den heiligen Quellen wirst du schattige Kühle finden; auf dieser Seite wird dir, wie immer schon, das Weidengebüsch an der Grenze zum Nachbarn, welches seine Blüten hybläischen Bienen als Weide bietet, ${ }^{17}$ oft durch leises Summen zum Schlaf einladen; auf dieser Seite wird unter einem hohen Fels der Baumscherer zu den Lüften singen und dennoch wird in der Zwischenzeit weder die schrill singende Ringeltaube, deine ganze Sorge, noch die Turteltaube aufhören, von der luftigen Ulme zu seufzen.

Tityrus wird hier also als Greis beschrieben, der zwar ein eher ärmliches Stück Land besitzt (1,47-48), welches ihm jedoch genug ist (et tibi magna satis; 1,47). Trotz der Ärmlichkeit wird sein Landgut, wie bereits gesagt, als locus amoenus beschrieben. Er hält in erster Linie Schafe und Rinder (1,8-9), aber offenbar auch Tauben (tua cura; 1,57-58). Ob er auch Bienen züchtet, wird nicht ganz klar. Es könnte sich bei den erwähnten Bienen sowohl um die des Tityrus als auch um die seines Nachbarn als auch um wilde Schwärme handeln. Die Bienen selbst tragen darüber hinaus eher als Element des locus amoenus zur Ausgestaltung dieses Gesamtbildes bei und sind nicht als aktiv handelnde Subjekte angesprochen. ${ }^{18}$ Dies zeigt sich bereits in der komplizierten Syntax: Auf grammatikalischer Ebene sind es gar nicht die Bienen selbst, die Tityrus zum Schlaf verleiten, sondern die saepes. Schon öfter ist in der Forschung beobachtet worden, dass Vergil an dieser Stelle vermutlich auf eine Passage in Theokrits 7. Eidyllion (135-147) verweist, wo das Land des Phrasidamos als locus amoenus geschildert wird, in dem Bienen

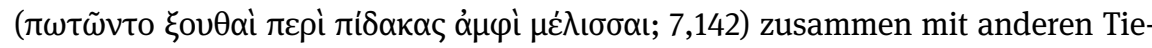
ren, Pflanzen und Landschaftselementen Teil der viele Sinne ansprechenden Inszenierung sind. ${ }^{19}$ Auch hier wird jedoch die Bienenzucht nicht eigens thematisiert und Phrasidamos zudem nicht weiter charakterisiert.

Der locus classicus für das Motiv eines älteren Bienenhalters ist sicherlich Vergils senex Corycius im vierten Buch der Georgica. Meist hat man sich in der bisherigen Forschung mit der Stellung und Bedeutung dieser Passage werkimmanent befasst und ist wenig auf mögliche Vorläufer und Nachfolger eingegangen..$^{20}$ Allgemein wird aber angenommen, dass Vergil hier auf Varros Darstellung der Ge-

17 Zur lateinischen Konstruktion vgl. auch Coleman (1977) 83-84; Clausen (1994) 52.

18 Vgl. z.B. Putnam (1970) 50; Olbertz (2008) 103 mit Anm. 57.

19 Vgl. z.B. Coleman (1977) 83; Thomas (1992) 52-53; Hunter (1999) 191; 193. Cucchiarelli (2012) 158-159 merkt an, dass die „hybläischen Bienen“ auch auf die neue theokriteische Art der Dichtung Vergils verweisen könnten.

20 Nur beispielhaft sei neben den einschlägigen Kommentaren auf folgende Arbeiten verwiesen: Burck (1956); La Penna (1977); Perkell (1981); Clay (1989); Thomas (1992); Thibodeau (2001); Monreal (2005). Letztere bietet auch eine gute Übersicht (6-12) über verschiedene Forschungsansätze. 
brüder Veianii reagiert, ${ }^{21}$ doch sind sicherlich auch andere Einflüsse zu erkennen, wie etwa Darstellungen der prinzipiell ähnlichen Figur des alten Mannes im Garten $^{22}$ oder, wie es Burck vermutet, auch von der bereits besprochenen Stelle in Ciceros Cato maior. ${ }^{23}$

Der korykische Greis wird folgendermaßen von Vergil innerhalb des noch etwas umfangreicheren Exkurses (4,116-148) vorgestellt (4,125-133; 139-141):

\footnotetext{
Namque sub Oebaliae memini me turribus arcis, qua niger umectat flaventia culta Galaesus, Corycium vidisse senem, cui pauca relicti iugera ruris erant, nec fertilis illa iuvencis nec pecori opportuna seges nec commoda Baccho. Hic rarum tamen in dumis olus albaque circum lilia verbenasque premens vescumque papaver regum aequabat opes animis seraque revertens nocte domum dapibus mensas onerabat inemptis.

$\cdots$

Ergo apibus fetis idem atque examine multo primus abundare et spumantia cogere pressis mella favis. ...
}

125

140

Und ich erinnere mich nämlich, dass ich unter den Türmen der oebalischen Burg (= Tarent), wo der dunkle Galaesus die gelblichen Fluren benetzt, einen korykischen Greis gesehen habe, der wenige Morgen unbebauten Landes besaß, und jenes nicht fruchtbar war durch (Pflug-)Stiere, ${ }^{24}$ noch ein geeignetes Feld für Vieh noch günstig für den Bacchus (= Weinbau). Indem er hier jedoch verstreut im Gebüsch Kohl pflanzte und umher weiße Lilien, Eisenkraut und mageren Mohn, setzte er die Reichtümer von Königen in seinem Geist gleich und überhäufte, wenn er spät in der Nacht nach Hause zurückkehrte, den Tisch mit ungekauften Speisen.

...

Also hatte er als erster einen Überfluss an fruchtbaren Bienen und einem großen Schwarm und gewann schäumenden Honig aus den gepressten Waben. ...

21 Vgl. z.B. Burck (1956) 164; Wilkinson (1969) 103-104; Tilly (1973) 299; La Penna (1977) 55; Salvatore (1977) 104-105; Perkell (1981) 172 Anm. 21; Mynors (1990) 274; Erren (2003) 824; Monreal (2005) 17-18. Teilweise (z.B. Burck; Wilkinson; La Penna) wird die Ansicht vertreten, dass memini me ... vidisse wörtlich zu verstehen sei und Vergil hier von einem realen Erlebnis berichte, das er dann nach literarischen Vorbildern überformt habe. Es gibt aber keinen Grund, eine biographistische Interpretation anzunehmen.

22 Vgl. Thibodeau (2001). Thomas (1992) schlägt auch Philitas als möglichen Vorläufer vor.

23 Vgl. Burck (1956) 165. De Saint-Denis' (1938) 308-311 These, dass Vergils Anlage der Georgica ganz auf diesen Satz zurückzuführen sei, geht aber sicherlich zu weit. Vgl. auch Powell (1988) 217. 24 Dieser Ausdruck bezieht sich wohl auf den Ackerbau, weil hier vermutlich alle traditionellen Bereiche der Landwirtschaft (Ackerbau, Viehzucht, Weinbau) genannt werden sollen. So z.B. Thomas (1988) 171; anders aber Erren (2003) 832-833. 
Einige Verbindungen lassen sich zur Figur des Tityrus in der ersten Ekloge ausmachen. ${ }^{25}$ Es handelt sich um ältere Männer, die über ein an und für sich nicht reiches Landgut verfügen, aber dennoch zufrieden damit sind. Das Land liegt jeweils an einem Fluss und Bäume spenden Schatten (siehe georg. 4,146). Ein offensichtlicher Unterschied, der aber nicht zuletzt dem literarischen Genre geschuldet ist, besteht in der Tätigkeit des Tityrus als Viehhirte.

In einigen Punkten entsprechen sich auch die Beschreibungen des korykischen Greises und der Gebrüder Veianii: Ihnen steht nur ein kleines Stück Land zur Verfügung, das sich kaum zur Landwirtschaft eignet. Die Lösung besteht in beiden Fällen im Anlegen eines Gartens, der unter anderem die Bienenzucht ermöglicht. Während es die Gebrüder Veianii jedoch damit zu einem gewissen materiellen Wohlstand gebracht haben, ist der Tisch des korykischen Greises zwar reichlich gedeckt, doch sind all diese Speisen nicht gekauft, d.h. selbst geerntet. Sein Reichtum ist explizit nicht finanzieller Art, dennoch setzt er seine Habe im Geist denen von Königen gleich (regum aequabat opes animis). ${ }^{26}$ Dies bedeutet wohl, dass die Darstellung Vergils als ein idealisiertes Bild eines durch das Wirken des Greises überreichen Gartens, der zu jeder Jahreszeit alles Notwendige bereitstellt, anzusehen ist. ${ }^{27}$

Die Charakterisierung des Greises und speziell sogar die letztgenannte Aussage zum Reichtum hat in der Forschung eine rege Diskussion hervorgerufen, ob man in dem Greis etwa das Idealbild eines epikureischen (oder auch stoischen) Weisen erkennen soll, der zurückgezogen lebt und mit dem zufrieden ist, was er hat. Dies muss jedoch offen bleiben. ${ }^{28}$ Dabei ist außerdem zu beachten, dass der Greis nicht völlig isoliert lebt, da das Wort potantibus $(4,146)$ auf mögliche Freun-

25 Vgl. dazu Thomas (1992) 53-54; Gale (2006) 180-181. Monreal (2005) 18-19 weist auf die interessante Tatsache hin, dass ein Vorgebirge einer Landzunge im Westen Kretas ebenfalls als Korykos bekannt war und es in der Nähe eine weitere Landzunge namens Tityros gab. Möglicherweise wollte Vergil durch die Namensgebung also selbst einen gelehrten Verweis auf die 1. Ekloge geben. 26 Vgl. z.B. Burck (1956) 161; La Penna (1977) 63. Der korykische Greis steht damit auch im Gegensatz zu den Darstellungen bei Aristophanes und Cicero. Zu weiteren Unterschieden in der Darstellung des älteren Cato bei Cicero und dem korykischen Greis vgl. auch Burck (1956) 166. Eine interessante Parallele könnte im Gleichnis vom Bienenhalter in Lucan. 9,283-293 enthalten sein. Dem pastor gelingt es hier, seine zunächst ausgeschwärmten Bienen zurückzurufen, und er freut sich, seiner (bescheidenen) Hütte den Reichtum bewahrt zu haben (divitias servasse casae ...; 9,292).

27 Vgl. auch Perkell (1981); Erren (2003) 825. Thomas (1988) 167 und vor allem Monreal (2005) 1215 sprechen sich dagegen dafür aus, dass das Bild des Gärtners „,realistisch“ sei, was nicht bedeute, dass sie von einer real existierenden Person als Vorlage ausgehen.

28 Vgl. z.B. La Penna (1977) 60-61; Erren (2003) 833-834. Gegen die Vereinnahmung einer bestimmten Philosophenschule z.B. Burck (1956) 170-171; Klingner (1967) 309-310 und Biotti (1994) 131. Gegen jedes Insinuieren einer philosophischen Haltung z. B. Monreal (2005) 16. Vgl. auch Gale (2006) 182. 
de hinweist, die mit ihm trinken. ${ }^{29}$ Eine Familie scheint er jedoch nicht $\mathrm{zu}$ haben, zumindest ist darüber keine Aussage getroffen. ${ }^{30}$ Letzteres ist auch immer wieder ein Ansatzpunkt für die von Servius (georg. 4,127) eingebrachte These, dass der Greis ein aus Korykos ${ }^{31}$ in Kilikien stammender ehemaliger Pirat sei, den Pompeius in der Nähe von Tarent angesiedelt habe. Das erscheint freilich spekulativ, hat aber in der Folge nicht nur zu einer umfangreichen Forschungsdebatte zu Servius' Behauptung geführt, ${ }^{32}$ sondern auch zu literarischen Auseinandersetzungen mit dem Thema etwa in dem neulateinischen Gedicht Senex Corycius (1902) von Giovanni Pascoli (1855-1912). ${ }^{33}$

Auch wird thematisiert, ob der Greis in seinem Verhalten den Bienen entspricht oder nicht. ${ }^{34}$ Insbesondere die englischsprachige Forschung sieht in dieser Passage einen Kontrast zur Gesellschaft der Bienen, der sich nicht nur an dem Alleinsein des Greises, ${ }^{35}$ sondern vor allem an der Frage nach dem labor des senex festmacht. ${ }^{36}$ Während in den Beschreibungen der Tätigkeiten des agricola und auch der Bienen das Wort labor öfter von Vergil gebraucht wird, taucht es in dieser Passage gerade nicht auf. Der Gartenbau wird mit cura bezeichnet. Zweifellos ist aber auch der korykische Greis das ganze Jahr über tätig, sodass man dem Fehlen des speziellen Begriffes dafür vielleicht nicht zu viel Bedeutung beimessen sollte. ${ }^{37}$ Zudem betont zumindest die Fachschriftstellerei, dass man sich stets um den Bienenstock zu kümmern habe. ${ }^{38}$

Wie auch in der Schilderung des Landguts in der 1. Ekloge steht auch in der des korykischen Greises die Bienenzucht nicht im Vordergrund, obgleich der Exkurs in dem der Bienenzucht gewidmeten vierten Buch der Georgica enthalten ist. Sie ist nur eine der zahlreichen Tätigkeiten des Greises, die sich ansonsten haupt-

29 Vgl. z.B. Gale (2006) 181.

30 Monreal (2005) 15 möchte daher nicht ausschließen, dass der Greis eine Familie besitzt, doch scheint die Schilderung dies zumindest nicht nahe zu legen.

31 Das Adjektiv Corycius könnte sich auf diese Stadt beziehen, auf die korykische Höhle am Berg Parnass, an dem die Musen leben, oder auch auf eine Landzunge auf Kreta. Vgl. z.B. Thomas (1988) 170-171; Thomas (1992) 61-62; Erren (2003) 831-832; Monreal (2005) 8.

32 Vgl. z B. Wilkinson (1969) 174-175; Thomas (1988) 170; Gale (2006) 182; Thibodeau (2001) 185186; Erren (2003) 831-832; Monreal (2005) 8.

33 Zur Rezeption des korykischen Greises in der neulateinischen Literatur vgl. Monreal (2005).

34 Dafür: z.B. Burck (1956) 170; Cramer (1998) 228; dagegen: z. B. Perkell (1981) 176; Clay (1989); Gale (2006) 182 (vgl. auch ebd. 180 mit Anm. 105).

35 Vgl. z.B. Clay (1989) 185.

36 Vgl. z.B. Perkell (1981) 168; Gale (2006) 180; 182.

37 Vgl. auch Burck (1956) 158-159; Gale (2006) 180.

38 Z.B. Colum. 9,9,1: Semper quidem custos sedule circumire debet alvaria, neque enim ullum tempus est quo non curam desiderent. Hier ist die beständige Tätigkeit interessanterweise als cura bezeichnet. 
sächlich auf den Gartenbau beziehen. Insofern ähnelt diese Passage mit einem alten Bienenzüchter eher den Stellen bei Aristophanes und Cicero und unterscheidet sich von Varros Bericht über die Gebrüder Veianii, bei denen die Bienenzucht im Fokus ihrer Tätigkeiten steht.

\section{Anthologia Graeca}

Eine dem korykischen Greis ganz ähnliche Figur findet sich auch in einem Epigramm des Apollonides (1. Jhd. n.Chr.) in der Anthologia Graeca (6,239 = III G./ P.): ${ }^{39}$

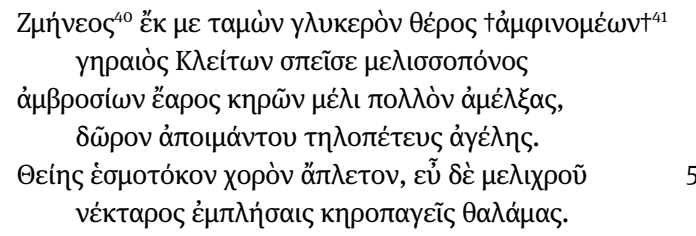

Aus der Wabe hat mich als süße Ernte geschnitten $<>$ und als Spende dargebracht der alte Imker Kleiton, nachdem er aus dem göttlichen Wachs des Frühlings viel Honig gemolken hat, als Gabe seiner hirtenlosen fernfliegenden Herde. Mach du seinen schwarmgebärenden Chor zahlreich, reichlich fülle die mit Wachs verschlossenen Zellen mit honigsüßem Nektar.

39 Für die folgenden Ausführungen zu Apollonides und die Übersetzung vgl. Berrens (im Druck). 40 Aufgrund der alphabetischen Ordnung der Gedichte nach dem ersten Wort, ist die Schreibung

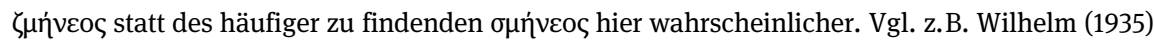
451-452; Merkelbach (1967); Gow/Page (1968 II) 150; Pelliccio (2013) 94. Vgl. auch Gutzwiller (1998) 37-39 zu Alphabetisierungen in Gedichtsammlungen, besonders in der des Philipp von Thessalonike. In der Suda ( $\sigma 734$ s. v. $\sigma \mu \eta \dot{v \eta}$ ) findet sich allerdings $\sigma \mu \eta \dot{v} v \varepsilon o \varsigma$, das Waltz (1931) 123 und Beckby (1965 I) 586 übernehmen.

41 Die Lesart hier entspricht der bei Gow/Page (1968 I) 128. Für diese Stelle wurden verschiedene Konjekturen vorgeschlagen: Dübner (1871) 253; Waltz (1931) 123 und Beckby (1965 I) 586 folgen

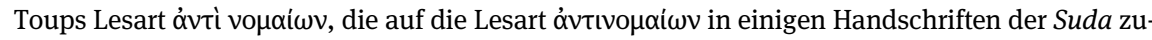
rückgeht. Dies übersetzen Waltz und Beckby mit ,au lieu des libations rituelles“ bzw. ,anstatt üblicher Spende“. Dies erscheint aber schwierig, da kaum erklärbar ist, warum Honig speziell für einen Imker keine übliche Spende sein soll (vgl. auch Gow/Page 1968 II, 150; Pelliccio 2013, 95). Besser wäre da schon Dübners Interpretation als „loco pecuniarum victimarum“. Da in dem Epigramm der Name der Gottheit fehlt, der die Gabe dargebracht wird, finden sich auch Konjekturen, die hier Pan oder einen Beinamen (z.B. vó $\mu$ ı૬) restituieren. Vgl. z.B. Dübner (1871) 253; Wilhelm (1935) 451; Gow/Page (1968 II) 150; Pelliccio (2013) 92-93; 95-96. 
Die Figur des Imkers Kleiton ist als yпpaı́s charakterisiert, der einer hier ungenannten Gottheit den Frühjahrshonig darbringt. ${ }^{42}$ Besonders interessant ist in diesem Gedicht die Verwendung von Begriffen aus der Fachsprache der Landwirtschaft. Es ist auffällig, dass Begriffe aus der Imkersprache in einigen Fällen durch solche aus anderen Bereichen der Landwirtschaft ersetzt wurden. So wird für die

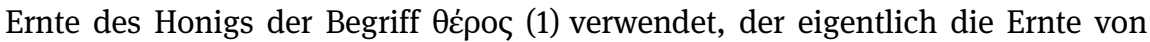
Getreide im Sommer bezeichnet. Ein Terminus technicus für das Ernten des Ho-

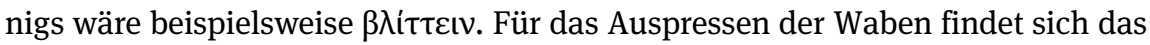
Verb $\alpha \mu \varepsilon \dot{\lambda} y \varepsilon ı v(3),{ }^{43}$ das zwar durchaus auch allgemein für das Auspressen einer Flüssigkeit verwendet werden kann, ${ }^{44}$ wörtlich aber ,melken“ bedeutet. Der

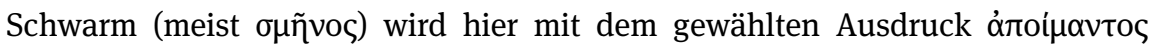

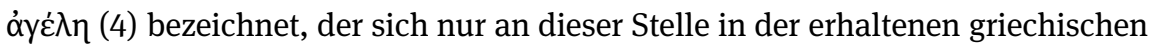
Literatur findet. ${ }^{45}$ Eine naheliegende Erklärung für dieses Phänomen könnte nun sein, dass durch diese Wortwahl auf knappem Raum ein ähnliches Bild gezeichnet werden soll, wie es uns bei Varro und besonders auch beim korykischen Greis in den Georgica begegnet: Kleiton stehen diese traditionellen Bereiche der Landwirtschaft, also Ackerbau, Milchvieh- und Weidewirtschaft, nicht zur Verfügung, sodass die Bienenzucht als Ersatz für diese dient.

Darüber hinaus gilt es noch festzuhalten, dass die Bienen in diesem Gedicht nun als eigenständige Akteure auftreten. Nicht nur stammt das Geschenk ( $\delta \tilde{\omega} \rho o v$;

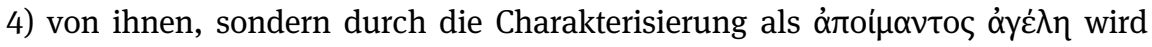
auch ihre - im Vergleich zu anderen Tieren - selbstständige Lebensweise hervorgehoben. Die Bienen sind somit nicht bloßes Beiwerk, sondern wichtige Akteure in diesem Epigramm.

Das Epigramm des Apollonides wurde von Philipp von Thessalonike in seine Sammlung aufgenommen. Letzterer hat auch ein eigenes Gedicht verfasst, das am Rande ebenfalls einen älteren Bienenzüchter erwähnt (Anthologia Graeca 9,438):

42 In der Antike wurde bis zu dreimal im Jahr Honig gewonnen. Der Honig von den Frühjahrsblüten galt dabei als der Beste. Z.B. Aristot. hist. an. IX 40, 626 b 28-31; Plin. nat. 11,34. Vgl. auch Pelliccio (2013) 98.

43 Dies ist in der Antike die übliche Vorgehensweise zur Gewinnung des Honigs. Die Waben wurden dabei zerstört; so z. B. Colum. 9,15,13; 9,16,1; Pall. agric. 7,7,3. Vgl. auch Crane (1999) 202.

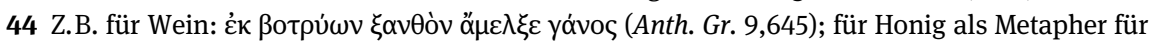

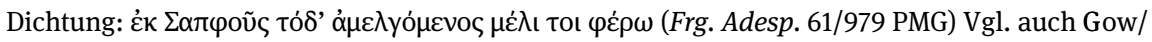
Page (1968 II) 150; Pelliccio (2013) 98-99.

45 Beim Adjektiv órлоí $\alpha$ vтоৎ (,ohne Hirte“) handelt es sich um ein Hapax legomenon (vgl. Gow/

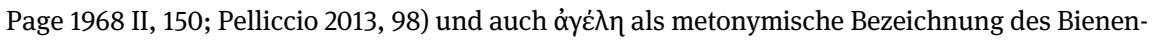
schwarmes scheint in der erhaltenen Literatur nur an dieser Stelle zu finden zu sein; abgesehen von eher entfernteren Beziehungen wie etwa der Definition der $\zeta \tilde{\omega} \alpha$ ко

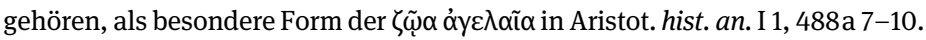




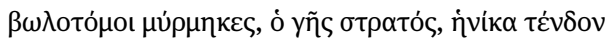

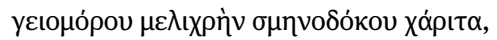

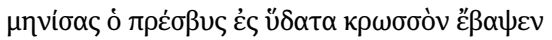

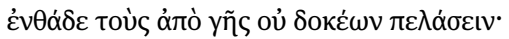

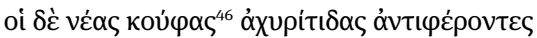

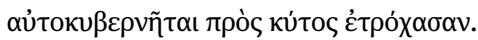

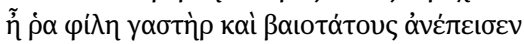

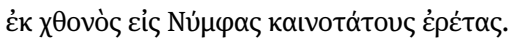

Als die erdschollenspaltenden Ameisen, die Armee der Erde, an den honigsüßen Gaben des bienenhaltenden Landbesitzers nagten, tauchte der erzürnte Alte den Krug ins Wasser, in der Meinung, dass diese nicht von der Erde dorthin gelangen werden; diese aber nahmen dagegen Strohhalme als unbeladene Schiffe und segelten selbst eilig auf das Gefäß zu. Wahrlich, der geliebte Magen hat so auch die Kleinsten dazu verleitet, sich als ganz neue Ruderer vom Land zu den (Meeres-)Nymphen (zu begeben).

Das Epigramm dient nun weniger dazu, den Imker als solchen zu charakterisieren, vielmehr liegt der Fokus ganz auf der besonders geschickten militärischen Leistung der Ameisen. Gerade dies zeigt jedoch, dass die Charakterisierung des

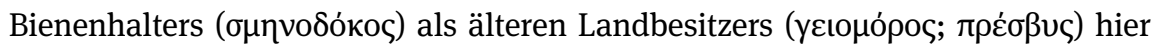
wohl topisch verwendet wird, weil sie für die Geschichte nicht zentral ist.

\section{Plinius, Naturalis historia}

Nicht nur in literarischen Texten im engeren Sinne, sondern auch in der Naturalis historia des Plinius lassen sich ähnliche Figuren finden. So verweist Plinius im Rahmen der Diskussion über die Fortpflanzung und Entwicklung der Bienen auf einen Konsular, der wohl aus wissenschaftlichem Interesse eine Art gläsernen Stock errichtet habe, um das Verhalten der Tiere beobachten zu können (nat. 11,49):47 Spectatum hoc Romae consularis cuiusdam suburbano alvis cornu lanternae tralucido factis („Man hat dies in Rom auf dem Landgut eines gewissen Kon-

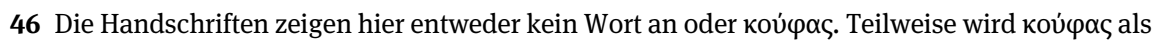
fehlerhaft angesehen und daher entweder durch Bruncks Konjektur kó $\varphi \rho \alpha \varsigma$ ersetzt (so Beckby 1965 III, 274 und Waltz/Soury 1974, 43) oder eine Lacuna offengelassen (so Gow/Page 1968 II, 358, die eher ein Adverb wie $\delta 0 \lambda i \omega \varsigma$ vermuten). Moore-Blunt (1977) 353-354 argumentiert aber mit guten Gründen für die Beibehaltung des überlieferten кoú $\alpha$ s.

47 Aus welchem Material ein solcher Stock tatsächlich bestand, ist nicht ganz einfach zu rekonstruieren. Hier scheint Plinius von dünn geschnittenem Horn auszugehen. An der zweiten Stelle (s. im Folgenden) der Naturalis historia ist jedoch vom lapis specularis, dem sogenannten Marienglas, die Rede. Vgl. König/Winkler (1999) 279-280. 
sulars mithilfe von Bienenstöcken beobachtet, die aus dem durchsichtigen Horn für Laternen hergestellt waren.“). Im 21. Buch über Heilpflanzen aus dem Garten ist ebenfalls von durchsichtigen Stöcken die Rede, die sich viele Leute in ihrem Garten aufstellen ließen (nat. 21,80): multi et e speculari lapide fecere, ut operantes intus spectarent (,Viele haben ihn [den Bienenstock] auch aus Marienglas hergestellt, um sie darin beim Arbeiten zu beobachten.“). In diesen Berichten lassen sich Parallelen zu den eher literarischen Texten ausmachen. Der sicherlich schon ältere Konsular und die übrigen Besitzer eines gläsernen Bienenstockes scheinen ihren Tieren eine gewisse Zuneigung und vor allem ein nicht nur ökonomisches Interesse entgegenzubringen, sodass sie sogar in einen solchen Stock investieren, um das Treiben ihrer Tiere beobachten zu können.

Darüber hinaus berichtet Plinius (nat. 11,19) von Aristomachos von Soloi und Philiskos von Thasos, zwei seiner - vermutlich hellenistischen - Quellen, die ihren Bienen besonders angetan gewesen sein sollen (ne quis miretur amore earum [sc. apium] captos ...). Aristomachos soll sich 58 Jahre lang mit nichts anderem als der Bienenzucht beschäftigt haben und Philiskos soll sich gar mit seinen Bienen in die Einsamkeit (in desertis) zurückgezogen und daher den Beinamen Agrius („der Wilde“) erhalten haben. In diesen Berichten von historischen Personen lassen sich ebenfalls Parallelen zum Motiv des alten Mannes erkennen, der in der Abgeschiedenheit von und mit seinen Bienen lebt.

\section{Ps.-Quintilian, Declamatio 13}

Der Verlust des geliebten Bienenschwarmes ist das Thema einer pseudo-quintilianischen Deklamation, die auch Apes pauperis („Die Bienen des armen Mannes“) genannt wird. Wie bei allen größeren Deklamationen ist die Autorschaft und die Abfassungszeit nur schwer $\mathrm{zu}$ ermitteln, allgemein geht man aber davon aus, dass das Corpus, welches sicherlich auf mehrere Autoren zurückgeht, im 2. Jhd. n. Chr. entstanden ist, unsere Declamatio eventuell auch etwas früher. ${ }^{48}$ Der in der Forschung bisher wenig beachtete Text ist die Anklagerede eines armen Mannes, der in der Nachbarschaft eines Reichen lebt. ${ }^{49}$ Der Arme hat auf seinem kleinen Stück Land Bienen gehalten, die aber auf den Blumen im Garten des Reichen weideten. Da der Reiche dies nicht dulden wollte, hat er die Blumen mit Gift benetzt, sodass die Bienen alle starben und der Arme somit seine Lebensgrundlage verlor.

48 So z.B. Ritter (1881) 58, dem sich auch Krapinger (2005) 24 im Allgemeinen anschließt. Zur Frage nach Verfasser und Entstehungszeit der größeren Deklamationen vgl. Håkanson (1986) 2284-2290. Vgl. Mantovani (2007) 374-379 für eine mögliche Verbindung zu Celsus.

49 Eine Übersicht über die Forschung bietet Krapinger (2005) 22-24. Dazu auch Mantovani (2007). 
Daher verklagt der Arme nun den Reichen. Bei der Rede handelt es sich also um eine Spielart des häufig in den Deklamationen der Kaiserzeit zu findenden Themas „Arm gegen Reich“.50

Die Deklamation selbst enthält viele eher juristische Passagen, ${ }^{51}$ doch bietet sie gerade in der narratio am Anfang sowie in der peroratio am Ende der Rede auch für unsere Fragestellungen interessante Charakterisierungen der Bienen und des Imkers sowie ihrer Beziehung zueinander.52 Vor allem diese Teile sind stark von den Georgica geprägt, was sich etwa in einer ähnlichen Wortwahl zeigt..$^{53}$ Parallelen finden sich nicht nur zum Exkurs des korykischen Greises, sondern aufgrund der Thematik auch zum sogenannten Aristaeus-Epyllion am Ende des 4. Buches von Vergils Georgica. ${ }^{54}$ Aristaios, der Sohn des Apollon und der Nymphe Kyrene, galt in der Antike als der Gründungsheros der Bienenzucht. Vergil berichtet von Aristaios' Verlust seines Bienenschwarmes als Folge seiner illegitimen Nachstellung der Eurydike. Aristaios hat also gewissermaßen Schuld am Verlust seiner Bienen, der Arme in dieser Deklamation dagegen nicht. Darüber hinaus verliert Aristaios bei Vergil seine Bienen aufgrund von Krankheit und Hunger, nicht durch Gift (amissis, ut fama, apibus morboque fameque; Verg. georg. 4,318).

Sich selbst und ihre Lage beschreibt die Erzählinstanz folgendermaßen ([Quint.] decl. 13,2):

Est mihi paternus, iudices, agellus, sane angustus et pauper, non vitibus consitus, non frumentis ferax, non pascuis laetus; ieiunae modo glebae atque humilis thymi, et non late pauperi casae circumiecta possessio. Verum mihi vel hoc fuit gratissima, quod non fuit digna, quam dives concupisceret. In hoc ego vitae meae secreto remotus a tumultu civitatis ignobile aevum agere procul ab ambitu et omni maioris fortunae cupiditate constitui et, dum molesta lege naturae transiret aetas, vitam fallere. Hoc mihi parvulum terrae et humilis tugurii rusticum culmen aequitas animi regna fecerat, satisque divitiarum erat nihil amplius velle.

50 Vgl. ausführlich Tabacco (1978a) und (1979); Krapinger (2005) 67-68; Mantovani (2007) 374376.

51 In der Rede geht es vor allem auch um das Verhältnis von ius und natura, speziell um die Frage inwieweit Bienen wilde Tiere sind und überhaupt einen Besitz darstellen, an dem ein Schaden verübt werden kann. Vgl. dazu z.B. Mantovani (2007) und im Anschluss an ihn Citti (2015) 119-121; Berti (2015) 41-43.

52 Zur Einteilung der Rede vgl. z.B. Ritter (1881) 175-179; Tabacco (1978a) 46-47; Krapinger (2005) $25-28$.

53 Vgl. ausführlich Tabacco (1978b), die auch noch weitere Parallelen zu anderen lateinischen Texten ausmacht, sowie Mantovani (2007) 346-349; Berti (2015) 35-44.

54 Selbstverständlich finden sich auch Anleihen aus anderen Teilen der Georgica, vor allem aus dem 4. Buch. „Il declamatore contamini Virgilio con Virgilio“, wie es Tabacco (1978b) 204 ausdrückt. 


\begin{abstract}
Mir gehört ein Äckerchen, vom Vater geerbt, ihr Richter, ziemlich schmal und ärmlich, nicht mit Weinreben bepflanzt, nicht fruchtbar für Getreide, nicht üppig für Weiden; nur von magerem Boden und niedrigem Thymian und kein Grundstück, das die arme Hütte weithin umgibt. Aber mir war es besonders deshalb allerliebst, weil es nicht wert war, dass es ein Reicher begehrte. Ich habe beschlossen in dieser Abgeschiedenheit meines Daseins abseits vom Treiben der Bürgerschaft ein ruhmloses Leben zu führen, fern vom Streben nach Ämtern und jeder Gier nach einem größeren Vermögen und unbemerkt zu leben, bis die Lebenszeit durch das unliebsame Naturgesetz vorübergeht. Dieses kleine bisschen Erde und die bäuerliche Behausung einer bescheidenen Hütte hatte mir die Ausgeglichenheit meines Gemütes zu Königreichen gemacht und es war mir genug Reichtum, nichts darüber hinaus zu wollen.
\end{abstract}

Die Sprecherinstanz verfügt also über ein nur kleines ererbtes Stück Land, das für kaum eine Form von Landwirtschaft geeignet ist. Trotzdem habe sie mit harter Arbeit ein zurückgezogenes und zufriedenes Leben geführt. Diese Motive sind aus Varros Res rusticae und Vergils Georgica bereits gut bekannt. ${ }^{55}$ Besonders die Formulierung humilis tugurii rusticum culmen aequitas animi regna fecerat verweist auf den bekannten Ausdruck regum aequabat opes animis in Verg. georg. 4,132.56 In ihrem Alter sei der Sprecherinstanz die körperliche Arbeit zunehmend schwer gefallen, ${ }^{57}$ weshalb sie sich auf die Bienenzucht verlegt habe, ${ }^{58}$ zumal die Bienen als flugfähige Tiere in der Lage seien, auch außerhalb des nur kleinen Landstückes, das zudem inzwischen noch ganz vom Garten des Reichen umgeben war, nach Nahrung zu suchen (13,2-3):

Namque ego, iudices, dum fortius opus permisit aetas, terram manibus subegi, et difficultatem labore perdomui, et invito solo nonnihil tamen fecunditatis expressi. Cito labitur dies, et proclivis in pronum fertur aetas; abiere vires, census meus, defectaque labore senectus, magna pars mortis, nihil mihi reliquit nisi diligentiam. [3] Circumspicienti, quod conveniret opus invalidae senectutis curae, succurrebat sequi pecora, fetuque placidi gregis paupertatem tueri, sed ex omni parte circumiectus divitis ager vix tenuem ad gressus meos semitam dabat. Quid agimus? inquam, undique vallo divitiarum clusi sumus. Hinc hortuli locupletis, hinc arva, inde vineta, hinc saltus; nullus terra datur exitus. Quaeramus animal, quod volet.

55 Vgl. Tabacco (1978b) 201-205; Krapinger (2005) 73.

56 Vgl. Tabacco (1978b) 203.

57 Die Charakterisierung als alter und armer Mensch kann zudem dazu dienen, beim Publikum Mitleid zu erzeugen. Vgl. Tabacco (1978b) 199.

58 Dieser Gedanke findet sich auch in [Quint.] decl. 13,4: Dederam laboribus meis iustam senex missionem; habebam, quae pro me opus facerent. Auch in einem Epigramm des Antiphilos in Anth.

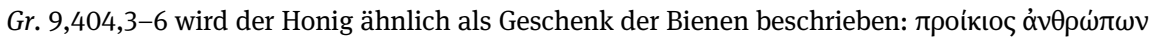

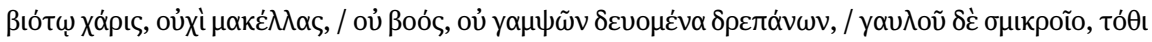

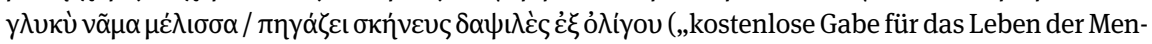
schen, die keiner Hacke, keines Ochsens, keiner gekrümmten Sichel bedarf, nur eines kleinen Eimers, wo eine Biene das süße Nass hervorsprudeln lässt, reichlich aus ihrem winzigen Körper."). 
Denn ich selbst habe, ihr Richter, so lange das Alter mir härtere Arbeit gestattete, die Erde mit meinen Händen bearbeitet, mit Mühe die Beschwerlichkeit bezwungen und holte aus dem unwilligen Boden doch manchen Ertrag. Schnell verrannen die Tage und das ansteigende Alter eilte dahin; es schwanden die Kräfte, mein Kapital, und geschwächt von der vielen Mühe war das hohe Alter, mehr tot als lebendig, und ließ mir nichts mehr als meine Sorgfalt. [3] Als ich mich umsah, welche Arbeit meiner Sorge um das kraftlose Alter passend wäre, kam mir der Gedanke, Vieh zu hüten und durch das Wachstum einer zahmen Herde mein ärmliches Leben zu bewahren. Aber der Acker des Reichen, der mich von allen Seiten umgab, bot mir kaum einen schmalen Fußpfad. „Was soll ich tun?“, sprach ich, „von allen Seiten sind wir vom Wall des Reichtums eingeschlossen. Hier die Gärtchen des Reichen, hier die Felder, dort die Weinberge, dort die Viehweiden; zu Lande gibt es keinen Ausgang. Wir wollen also ein Tier suchen, das fliegt.“

Wenn man denn wollte, könnte man in der Aussage, dass die Bienen die Arbeit für den Imker erledigen, der dazu selbst nicht mehr in der Lage ist, eine frühe Vorwegnahme der oben angesprochenen Forschungsdiskussion zum labor des korykischen Greises sehen. Gewisse Ähnlichkeiten weist diese Aussage auch zu dem bereits besprochenen Epigramm des Apollonides auf. Dort (Anth. Gr. 6,239,4)

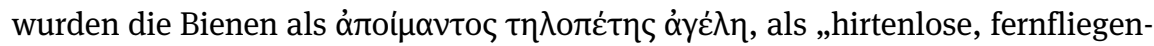
de Herde“ bezeichnet. Dies impliziert eine analoge Vorstellung, wie sie hier in der Declamatio begegnet: Die Bienen sind selbstständige Tiere, die keiner großen Aufsicht bedürfen, und sind durch ihre Fähigkeit zu fliegen nicht an die engen Grenzen eines kleinen Landgutes gebunden.

Ein wesentlicher Unterschied zu den Darstellungen bei Varro und Vergil liegt in der Begründung der Entscheidung zu Gartenbau und Bienenzucht. Während es in Varros und Vergils Darstellungen primär eine Entscheidung aufgrund der mangelnden Eignung des Landgutes für andere Formen der Landwirtschaft war, die den Ausschlag gab, ist der Fall hier ein wenig anders gelagert. Das Land des Armen ist zwar auch kaum ertragreich, doch konnte er mit viel Mühe, Ertrag aus dem Boden gewinnen. Erst als die schwindende Kraft im Alter keine andere Tätigkeit mehr erlaubte, habe er sich auf die Bienenzucht verlegt, nicht aber von Anfang an..$^{59}$ Das fortgeschrittene Lebensalter ist hier also kein zufälliges Attribut des Protagonisten, sondern wird als entscheidend für die Wahl der Bienenzucht angeführt. Ähnlich wie die Gebrüder Veianii bei Varro, aber im Gegensatz zum korykischen Greis bei Vergil, widmet sich der Arme nun ganz ausschließlich der Bienenzucht. ${ }^{60}$ Wie die Gebrüder Veianii lebt er vom Verkauf seiner Produkte in der Stadt $(13,3)$ und nicht autark von dem, was sein Garten hervorbringt. Der Bie-

59 Vgl. auch Tabacco (1978a) 56.

60 Vgl. auch Tabacco (1978b) 204-205 Anm. 28. Innerhalb der Declamatio spielt es dabei sicherlich auch eine Rolle, dass der Arme somit in genauem Gegensatz zum Reichen steht - ein wichtiges Strukturelement der Rede (vgl. Tabacco 1978a, 48-49). Der Reiche verfolgt nämlich die traditionel- 
nenzüchter dieser Declamatio bleibt aber arm, ${ }^{61}$ was in einem gewissen Widerspruch zur Darstellung in antiken Fachtexten steht, die die Bienenzucht meist als lukrativ beschreiben (s. Anm. 9), wenngleich letztere freilich aus der Sicht von Großgrundbesitzern geschrieben sind.

Gerade in Bezug auf sein ärmliches Leben vergleicht sich die Sprecherinstanz explizit mit seinen Bienen (13,3): nam quid apibus invenit natura praestantius? parcae, fideles, laboriosae. o animal simile pauperibus! („Denn was hat die Natur Hervorragenderes ersonnen als die Bienen? Sie sind sparsam, treu und arbeitsam. Oh du den Armen so ähnliches Tier!“). ${ }^{62}$ In den Eigenschaften der Sparsamkeit, der Treue und des Fleißes sind die Tiere also den armen Menschen vergleichbar. ${ }^{63}$ Zudem werden sie hier emphatisch direkt angesprochen, der Unterschied zwischen Mensch und Tier wird also nivelliert. Mehrmals wird in dieser Declamatio das treue Verhalten der Bienen betont $(13,3 ; 16 ; 17)$. Mensch und Tier stehen also in einem engen Verhältnis der fides. An mehreren Stellen berichtet die Erzählinstanz zudem, dass sie großen Schmerz über den Verlust der Tiere empfinde $(13,5 ; 7 ; 15)$ und geht sogar so weit zu behaupten, sie hätte selbst gerne das Gift genommen, wenn sie welches gefunden hätte (libenter bibissem, si invenissem, venenum; 13,5). Ausführlich und sehr emotional wird auch das Auffinden der toten Tiere geschildert (13,5-6). Die Vergiftung der Tiere wird darüber hinaus mit Kapitalverbrechen an Menschen gleichgesetzt (13,7; 14).

Bei aller Zuneigung zu den Bienen wird der Unterschied zwischen Mensch und Tier hier jedoch nicht völlig eingeebnet. Sie sind für die Sprecherinstanz auch Geschöpfe, die sie sich in ihrem Alter zu Nutzen machen kann (13,3-4; s.o.), und ganz deutlich sagt sie noch einmal gegen Ende der Rede, dass die Natur Tiere zum Nutzen für den Menschen geschaffen habe $(13,15),{ }^{64}$ die Bienen aber zusätzlich noch Freude bereiteten (nam ut cetera animalia videtur mihi natura usibus nostris genuisse, haec etiam deliciis; 13,15). Die Tiere sind also nicht nur die finanzielle Grundlage des Alten, sondern darüber hinaus eine wichtige soziale oder moralische Stütze, zumal der Sprecher nicht über eine Familie zu verfügen scheint und

len Formen der Landwirtschaft Gartenbau, Ackerbau, Weinbau und Viehzucht (13,3; s.o.), der Arme aber nur die Bienenzucht, die der Reiche nicht betreibt.

61 Bereits im ersten Satz der Declamatio $(13,1)$ sagt er über sich: ... iam ante quam quod habebam perdidi, pauper ... („Schon bevor ich alles verlor, was ich hatte, war ich arm.“).

62 Generell wird teilweise angenommen, dass Bienen den Reichtum hassen, vgl. Berrens (2018) 372.

63 Diese Charakteristiken finden sich typischerweise für arme Menschen in den Deklamationen. Vgl. Tabacco (1978a) 44.

64 Mantovani (2007) 355-359 erkennt darin eine stoische, anthropozentrische Haltung. Ihm folgt Citti (2015) 120. 
die Bienen ihm diese ersetzen. ${ }^{65}$ Dazu passt auch, dass er gleich zu Beginn der Declamatio betont, er sei weniger an der Ernte des Honigs und dem Verkauf in der Stadt interessiert, als an der Arbeit mit den Bienen (13,3). Nicht nur die finanzielle Grundlage, sondern gewissermaßen die Familie und den Grund zur Freude, hat der Reiche dem Armen durch die Tötung der Bienen genommen, was die Größe seines Verbrechens noch erhöht. ${ }^{66}$

\section{Aelian, Epistula rustica 5}

In einer der Epistulae rusticae Aelians ist ein weiteres interessantes Beispiel unseres Motivs, wiederum in Verbindung mit dem Verlust des Bienenschwarmes, erhalten. Das fiktive Milieu dieser Briefe ist die Welt attischer Bauern. ${ }^{67}$ Da Attika in der Antike berühmt für seinen exzellenten Honig war (s.o.), ist es nicht verwunderlich, dass auch ein Imker zu Wort kommt. ${ }^{68}$ In Brief 5 beklagt sich hier Baiton gegenüber Anthemion, dass ihn seine Bienen verlassen hätten.

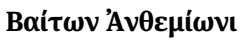

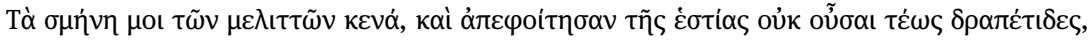

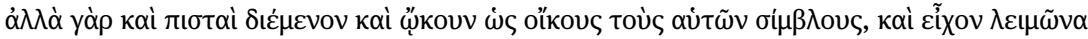

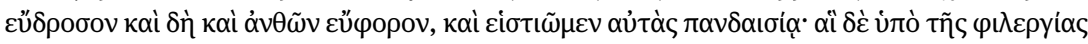

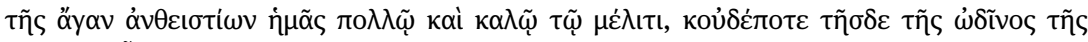

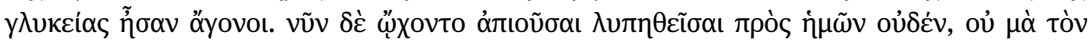

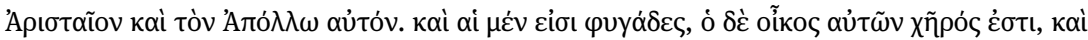

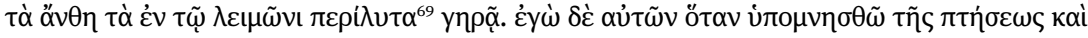

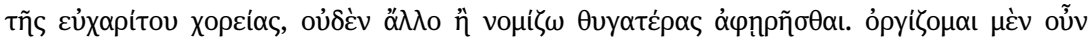

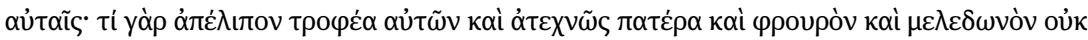

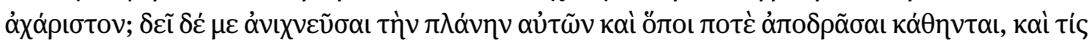

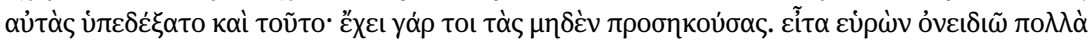

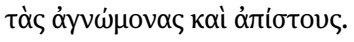

65 Vgl. Tabacco (1978a) 58.

66 Vgl. Tabacco (1978a) 50.

67 Vgl. Kindstrand (1998) 2978.

68 Auch unter den Bauernbriefen des Alkiphron findet sich die Erwähnung der Bienenzucht $(2,20)$. Das Motiv ist jedoch ein anderes, sodass Alkiphrons Brief hier nicht beachtet wird.

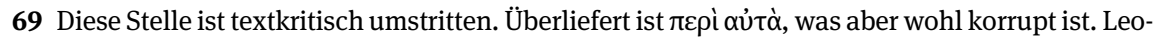
ne setzt es daher in Cruces. Das ursprünglich von Post vorgeschlagene $\pi \varepsilon \rho i \lambda u \tau \alpha$ wird sowohl von Benner/Fobes als auch von Domingo-Forasté übernommen. Eine ausführliche Diskussion findet sich bei Drago (2013) 319-323, die aufgrund einer möglichen Parallele bei Aristainetos als weitere

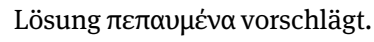




\section{Baiton grüßt den Anthemion}

In meinem Stock sind keine Bienen mehr. Sie haben ihr Heim verlassen, obwohl sie zuvor keine Ausreißer waren, sondern im Gegenteil treu dortblieben und ihre eigenen Stöcke bewohnten wie Häuser. Und ihre Wiese war stets taubedeckt und, ja, auch voll von Blumen und ich habe sie immer mit einem Mahl bewirtet, bei dem nichts fehlte. Diese aber haben mich aufgrund ihres das Normalmaß überschreitenden Fleißes im Gegenzug mit viel schönem Honig bewirtet und niemals mussten wir auf ihre süße Leibesfrucht verzichten. Nun aber sind sie weggegangen und verschwunden, obwohl sie niemals etwas von mir erleiden mussten, niemals, bei Aristaios und Apollon selbst. So sind sie nun entflohen, ihr Haus aber ist verwaist und die Blüten auf der Wiese verwelken ringsum. Jedes Mal, wenn ich aber an ihren Flug und ihren anmutigen Tanz denke, glaube ich nichts anderes, als dass mir meine Töchter geraubt worden seien. Ich bin ihnen aber nun böse. Denn warum haben sie ihren Ernährer und schlichtweg ihren Vater, Wächter und nicht undankbaren Kümmerer verlassen? Ich muss aber ihrem Umherirren nachspüren und sehen, wohin auch immer sie sich nach ihrem Fortgehen niedergelassen haben. Und, wer sie aufgenommen hat, auch dies: Denn er hat sie ja sicherlich, obwohl sie ihm nicht gehören. Wenn ich sie dann gefunden habe, werde ich sie heftig schimpfen, die Undankbaren und Treulosen.

Ähnlich wie der Arme in der Deklamation betont Baiton in dem Brief ganz explizit die enge Beziehung zu seinen Bienen, indem er ihren Verlust mit Töchtern ver-

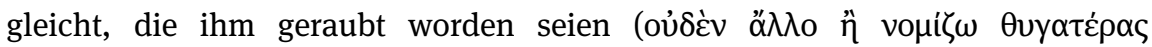
$\dot{\alpha} \varphi \eta \rho \tilde{n} \sigma \theta \alpha \mathrm{l})$, sowie sich selbst also ihren Ernährer, Vater, Wächter und Kümmerer

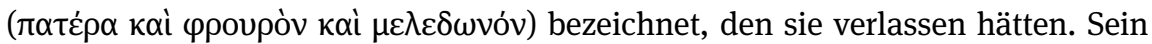

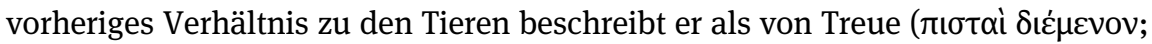

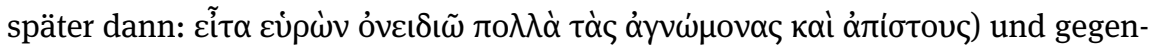
seitigem Nutzen geprägt. Man könnte hier fast von einer $\xi \varepsilon v i \alpha$ sprechen, die durch die Formen $\varepsilon i \sigma \tau \iota \tilde{\omega} \mu \varepsilon v$ und $\alpha \dot{v} \theta \varepsilon ı \tau \tau i \omega v$ ausgedrückt wird. ${ }^{70}$ Doch tritt ebenfalls an einigen Stellen ein etwas anderes Bild hervor, wenn Baiton die abgegangenen

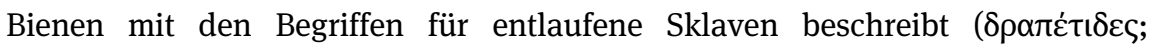
$\varphi v y \alpha ́ \delta \varepsilon \varsigma)$. Baiton ist jedoch davon überzeugt, dass er seinen Tieren niemals Unrecht getan habe, wie er bei Aristaios und Apollon schwört. In diesem Zusammenhang ist vor allem der erstgenannte Aristaios interessant. Die Anrufung des Gründungsheros der Bienenzucht ist generell passend in diesem Kontext, Leone, Drago und Smith weisen aber wohl zu Recht darauf hin, dass hier noch ein weiterer Aspekt bedeutsam ist: ${ }^{71}$ Aristaios selbst hat, wie bereits gesagt, ebenfalls seinen Bienenschwarm verloren. Baiton hält sich allerdings im Gegensatz zu Aristaios ausdrücklich für unschuldig und ist deshalb entsprechend erzürnt über seine Bienen, die ihn so treulos verlassen haben, und möchte sie heftig tadeln, wenn er sie wiedergefunden habe. Diese Aussage ist im letzten Satz des gesamten Briefes ent-

70 So Smith (2014) 38.

71 Vgl. Leone (1977) 64 Anm. 12; Drago (2013) 314 Anm. 7; Smith (2014) 38. 


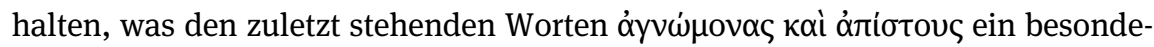
res Gewicht verleiht.

Kurzum, in Aelians Brief begegnet uns der Bienenzüchter in einer eher ungewohnten Rolle: In der des verschmähten Vaters oder Freundes, man könnte vielleicht auch Liebhabers sagen, ${ }^{72}$ dessen zuvor große Zuneigung in nun ebenso großen Zorn umgeschlagen ist. Eine starke Emotionalisierung der Tier-Mensch-Beziehung tritt hier zutage. Mensch und Tier stehen moralisch auf einer Ebene. ${ }^{73}$ Dies wird nicht zuletzt durch die Wahl der entsprechenden Termini ersichtlich, mit denen Baiton seine Beziehung zu den Bienen charakterisiert, als Vater-Töchter-

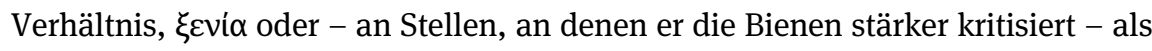
das von Herr und Sklaven. Besonders die beiden ersten Arten von Beziehungen basieren in hohem Maße auf Vertrauen und ein Bruch zählt zu den schlimmsten Freveln. Dadurch wird ersichtlich, auf welcher Ebene die Mensch-Tier-Beziehung hier angesiedelt ist, nämlich als inniges Vertrauensverhältnis unter Gleichen.

Dazu sollte man noch einen weiteren Punkt beachten. Nur sehr kurz, fast ganz am Ende des Briefes thematisiert Baiton, dass seine Bienen sich möglicherweise bei jemand anderem niedergelassen haben. Smith äußert die Theorie, dass der hier nicht namentlich Genannte der Adressat des Briefes, Anthemion, sei. ${ }^{74}$ Dies leitet er unter anderem aus der emphatischen Partikel to ab, die er hier eher in ihrer etymologisch ursprünglichen Bedeutung als ethischer Dativ von oú verstehen möchte. Diese Interpretation entbehrt nicht eines gewissen Charmes. Dies gilt insbesondere für das von Smith ebenfalls hervorgehobene Verständnis des Namens „Anthemion“, der auf öv $\theta$ oৎ zurückzuführen ist. ${ }^{75}$ Nur ein blumenreicher Mann sei also in der Lage, die Bienen des Baiton zu verführen. Diesem wird jedoch kaum Schuld gegeben, der moralische Fehler wird allein den Tieren angelastet, die das Vertrauensverhältnis beschädigt haben. Der Schmerz entsteht vor allem deshalb, weil das Verhalten nach Baitons Darstellung eigentlich nicht normal ist. Viele naturkundliche agronomische Schriften betonen allerdings, dass es üblich sei, dass zumindest ein Teil des Volkes im Frühsommer auszieht, wie es ja

72 Drago (2013) 315 macht in dem Brief eine erotische Lexik aus. Man könnte vielleicht einwenden, dass die Selbststilisierung Baitons als Vater und Kümmerer nicht so recht zu einer Rolle als Liebhaber passt. Drago verweist jedoch auf die berühmte Ansprache Andromaches an Hektor in der Ilias (6,429-430), in der sie sich wünscht, er solle ihr Vater, Mutter, Bruder und starker Ehemann

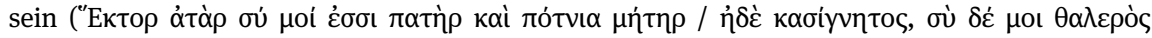

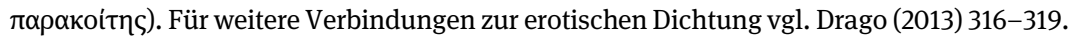

73 Diese enge, gleichwertige Beziehung zwischen Tier und Mensch ist ein Charakteristikum der Epistulae rusticae allgemein. Vgl. Smith (2014) 34; 36-41.

74 Vgl. Smith (2014) 38-39.

75 Dies findet sich auch bei Drago (2013) $311 \mathrm{Anm} .1$. 
auch tatsächlich der Fall ist. ${ }^{76}$ Ein natürliches Verhalten der Bienen wird durch die emotionale Beziehung, die Baiton $z u$ ihnen aufbaut, zu einer eher für einen Menschen verwerflichen Tat stilisiert. Das Lebensalter Baitons wird im Brief nicht explizit angesprochen, doch legt die Betonung eines Vater-Töchter-Verhältnisses nahe, dass er sich zumindest nicht als jungen Mann sieht. Möchte man Dragos Interpretation der Inszenierung Baitons als verschmähtem Liebhaber folgen, ${ }^{77}$ könnte man noch auf einen weiteren Aspekt hinweisen. Sollte die Figur des Imkers, wie es die übrigen Beispiele aus dieser Zeit nahelegen, tatsächlich primär als älterer Mann konzeptualisiert worden sein, so könnte man darin auch einen Grund für die Verschmähung durch seine Bienen erkennen: Sie könnten zu einem Mann gegangen sein, der - wie es sein Name nahelegt - noch in der Blüte seiner Jahre steht.

In diesem Zusammenhang gilt es auch den Charakter dieses Briefes und des gesamten Corpus zu beachten. Viele Briefe weisen eine Beeinflussung nicht nur durch die Bukolik, sondern auch durch die Komödie auf. ${ }^{78}$ Diese pathetisch überhöhte Klage über den Verlust der Tiere könnte somit ein Mittel der Ironie und der Komik sein. ${ }^{79}$

\section{Corpus Aesopicum, Fabula 72 (Perry $=74$ Hausrath)}

Für die unter dem Namen Aesops überlieferten Fabeln gilt allgemein, dass ihre genaue Herkunft und Abfassungszeit schwierig zu bestimmen ist. Erstmals in der sogenannten Collectio Augustana, die vermutlich im 2. Jhd. n.Chr. entstanden ist, ${ }^{80}$ nicht aber in den Sammlungen des Phaedrus und des Babrios, ist eine Fabel vom Imker und seinen Bienen enthalten: ${ }^{81}$

76 Z.B. Aristot. hist. an. IX 40, 625 b 6-17; Varro rust. 3,16,29; Verg. georg. 4,163-164; Colum. 9,9,1; 9,12,1; 9,14,5; Plin. nat. 11,54; Pall. agric. 7,7,4.

77 Vgl. Drago (2013).

78 Vgl. z.B. Benner/Fobes (1949) 345; Kindstrand (1998) 2980; Drago (2013) 314; Smith (2014) 32.

79 Vgl. Drago (2013) 316.

80 Vgl. z.B. Holzberg (2012) 83, der für eine Entstehung in der frühen Kaiserzeit plädiert.

81 Holzberg (2012) 100 betont, dass sich ,über die von ihrem (sc. der Collectio Augustana) Autor benutzten Vorlagen nichts Sicheres sagen“ ließe. Etwa die Hälfte der 231 Fabeln umfassenden Collectio Augustana findet sich nur dort (oder in späteren byzantinischen Sammlungen) und es ist nach Holzberg nicht unwahrscheinlich, dass diese Fabeln auf denjenigen zurückgehen, bei dem sie zum ersten Mal auftauchen. Nøjgaard (1964) 162; 193; 376 hebt hervor, dass Anlage und Erzählung der Fabel ungewöhnlich sind. 


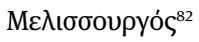

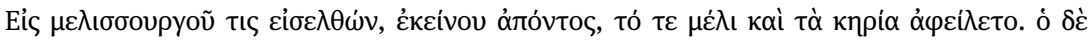

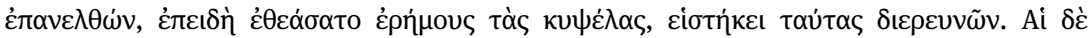

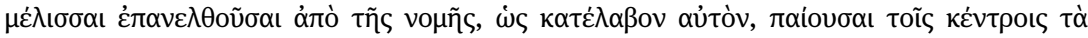

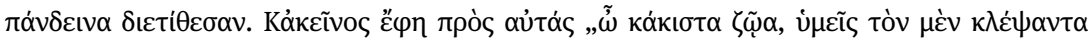

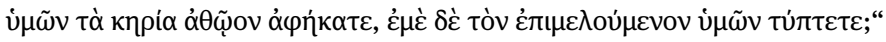

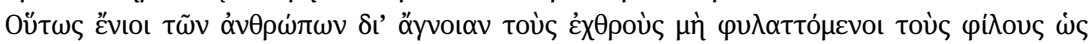

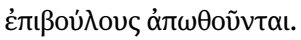

Es kam jemand zu einem Imker, als dieser nicht zuhause war, und stahl den Honig und die Waben. Als jener (sc. Imker) wieder zurückkehrte und sah, dass die Bienenstöcke leer waren, blieb er stehen und untersuchte sie. Wie nun aber die Bienen von der Weide zurückkamen und ihn dort antrafen, stachen sie ihn mit ihren Stacheln und richteten ihn übel zu. Und jener sagte zu ihnen: „Ihr schlechtesten Tiere. Ihr habt den, der euch bestohlen habt, unbestraft davonkommen lassen, mich aber, der ich mich um euch sorge, stecht ihr?“

So schützen sich manche Menschen aus Ignoranz nicht vor ihren Feinden und verstoßen ihre Freunde in der Annahme, sie wollten ihnen Übles tun.

Über den Imker selbst wird nicht viel berichtet, außer dass er sich nach eigener Aussage um seine Tiere sorgt. Wie es für eine Fabel nicht ungewöhnlich ist, kommt den Tieren eine prominente Rolle zu. Sie sind die direkten Ansprechpartner des Imkers, auch wenn Sie selbst keine Antwort geben, und ihr Verhalten wird an menschlichen Maßstäben gemessen. Ihr ignorantes und treuloses Vorgehen

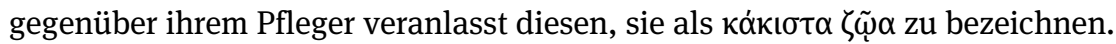

In seiner Enttäuschung und Entrüstung über das ignorante Verhalten der Bienen kann der Imker in dieser Fabel an Baiton in Aelians 5. Epistel erinnern. Gleichwohl unterscheiden sich die Geschichten in einigen Punkten, sodass man vermutlich nicht von einer gegenseitigen Beeinflussung ausgehen sollte. Dennoch ist die thematische und motivische Nähe der Epistel zur Fabel interessant.

\section{Zusammenfassung und Ausblick}

Es zeigt sich, dass die Figur des Bienenhalters in verschiedenen Gattungen der griechischen und lateinischen Literatur auftreten kann und vor allem in der Kaiserzeit an Kontur gewinnt. Während die Bienenzucht etwa in den Darstellungen bei Aristophanes, Cicero und auch bei Vergil nur eine unter vielen landwirtschaftlichen Tätigkeiten ist, lässt sich in den späteren Texten (und auch bereits bei Var-

82 Ich folge hier Perrys Text, der auf der Collectio Augustana beruht. Die Fabel ist auch in der Collectio Accursiana enthalten (ed. Hausrath 74), doch sind die Unterschiede ausschließlich sprachlicher, nicht inhaltlicher Natur. 
ro) eine stärkere Fokussierung auf das Leben mit speziell diesen Tieren erkennen. Dies geht auch mit einer deutlicheren Betonung der Handlungs- und Wirkungsmacht der Bienen einher. Während die Tiere etwa bei Aristophanes und in Vergils Eklogen eher als Staffage dienen, die das Landleben illustrieren, und sie bei Varro als geschickte Möglichkeit genannt werden, ein gutes Einkommen zu erzielen, so steht in Texten der Kaiserzeit dagegen die emotionale Beziehung des Bienenhalters zu seinen Bienen oftmals im Vordergrund, wenngleich die anderen Aspekte nicht völlig ausgeblendet werden. Bei allen Unterschieden im Detail kann man also vor allem in den kaiserzeitlichen Texten, den griechischen wie den lateinischen, eine Emotionalisierung der Tier-Mensch-Beziehung erkennen. Zudem werden die Tiere in stärkerem Maße als fast schon ebenbürtige Mitlebewesen wahrgenommen, denen wie dem Menschen bestimmte Rechte und Pflichten zuteilwerden. Dies wird gerade in den letztgenannten Beispielen, in der pseudoquintilianischen Deklamation, in Aelians Brief sowie - hier allerdings auch gattungsbedingt - der Fabel deutlich.

Diese Beobachtung stimmt mit der allgemeinen Entwicklung der zoologischen und (natur-)philosophischen Literatur überein. Seit dem Hellenismus, besonders aber in der Kaiserzeit lässt sich eine Abkehr von der aristotelischen und theophrastischen Naturforschung hin zu einer stärker anthropomorphisierenden und vor allem an wundersamen Verhaltensweisen interessierten Tierkunde erkennen. ${ }^{83}$ Insofern ist es verständlich, dass gerade in dieser Zeit auch den Bienen und ihrem Verhalten eine stärkere Rolle in der Literatur zukommt und sie als eigenständige Akteure und adäquate Gegenüber zu ihren Haltern auftreten. Dazu passt auch, dass nach Berichten des Plinius tatsächlich ein genuines Interesse an diesen Tieren bei einigen Gutsbesitzern entstand.

Während erste Ansätze des Motivs eines älteren Imkers auf seinem kleinen Landgut schon bei Aristophanes, Cicero und auch bei Varro zu erkennen sind, tritt es erstmals deutlich bei Vergil auf und findet sich dann als Topos in verschiedenen griechischen und lateinischen Texten der Kaiserzeit. Je nach Darstellungsabsicht und Gattung kann das Motiv jedoch angepasst und Elemente ausgelassen oder ergänzt werden. So findet sich die Erwähnung des Alters bei Cicero, Vergil, in den beiden Gedichten der Anthologia Graeca sowie in der pseudo-quintilianischen Deklamation (man könnte noch den Konsular und Aristomachos bei Plinius hinzunehmen), in der Epistel Aelians sowie in der Fabel wird es aber zumindest nicht explizit erwähnt. Nur in der Deklamation wird eine Begründung für die Verbindung von Alter und Bienenzucht angebracht: Diese Tätigkeit wird hier als für einen körperlich eingeschränkten Menschen gut geeignet angeführt, weil sie

83 Vgl. z.B. Lennox (1994); Kullmann (1998) v.a. 127-129; Föllinger (2003) v.a. 76-80. 
weniger anstrengend und mühevoll ist. In den übrigen Texten fehlt eine solche Begründung, sodass das Alter zu einem bloßen Attribut des Bienenhalters wird, jedoch möglicherweise mit der lebensweltlichen Erfahrung übereinstimmt.

Darüber hinaus sind die literarischen Beispiele, insbesondere die kaiserzeitlichen, größtenteils im kleinbäuerlichen Milieu zu verorten; das dürfte wohl auch für Aelians Epistel und die Fabel gelten, wenngleich die Bescheidenheit des Landgutes dort nicht so stark betont wird. Die Bienenzucht wird teilweise in Verbindung mit dem Anlegen eines dafür geeigneten Gartens als Möglichkeit für ein Stück Land eingeführt, das für keine andere Form von Landwirtschaft geeignet ist. Die Welt des Bienenzüchters ist dabei oftmals dezidiert von einer städtischen Gesellschaft abgetrennt.

Teilweise sind die Unterschiede in der Ausgestaltung der Figur auch durch die jeweils gewählte literarische Form bedingt. Der Fokus auf Tiere ist ein konstitutives Element der Fabel und auch typisch für Aelians Epistulae sowie für einige Epigramme. In anderen Gattungen ist dieser Aspekt jedoch weniger zentral. Ebenso kann man das Fehlen einer ausführlichen Beschreibung des Gartens, der im Zentrum von Vergils Exkurs steht, etwa in der Fabel, der Epistel oder den Epigrammen damit erklären, dass dies kleine Formen sind, die sich daher auf einen Aspekt konzentrieren und keine ausführliche Schilderung anderer Elemente geben können. Auch wenn der Imker oftmals arm ist, müssen die Gebrüder Veianii reich sein, weil es der Intention der Res rusticae entspricht, die Landwirtschaft als lukrativ darzustellen.

Es ist schwierig zu sagen, inwieweit Abhängigkeitsverhältnisse zwischen den einzelnen Texten bestehen. Sicherlich rekurriert Vergil auf Varro und die pseudoquintilianische Deklamation wiederum auf beide, vor allem aber auf Vergil. Auch wenn sich ein Bezug auf Vergil weniger deutlich ausmachen lässt, gilt sowohl für Apollonides und Philipp als auch für Aelian, dass sie mit lateinischer Literatur in Berührung gekommen sind und Vergils Texte daher vermutlich kannten. ${ }^{84}$

Die Verwendung des Bildes vom alten Mann mit seinen Bienen endet nicht in der Spätantike, sondern wird auch in der mittel- und neulateinischen Literatur immer wieder aufgenommen, hier meist dezidiert an Vergils korykischen Greis angelehnt. ${ }^{85}$ Beispielhaft sei nur auf die gewissermaßen konträre Gestalt des Mannes aus Viterbo in Marco Girolamo Vidas De Bombyce $(2,142-163)^{86}$ sowie auf Pascolis bereits erwähnten Senex Corycius hingewiesen. ${ }^{87}$

84 Vgl. Berrens (im Druck).

85 Vgl. z.B. Krapinger (2007) zur Rezeption der pseudo-quintilianischen Declamatio 13 im Mittelalter. Vgl. z.B. Haskell (1999); Haskell (2003) 11; 20; Monreal (2005) für neulateinische Literatur.

86 Dazu Monreal (2005) 29-30.

87 Vgl. dazu die Edition mit Einleitung und Kommentar von de Meo (1974). 


\section{Verzeichnis der verwendeten Literatur}

Claudii Aeliani Epistulae rusticae, ed. P. A. M. Leone, Mailand 1974.

Claudius Aelianus, Epistulae et fragmenta, ed. D. Domingo-Forasté, Stuttgart/Leipzig 1994.

Corpus fabularum Aesopicarum, vol. I, fasc. I, ed. A. Hausrath, Leipzig 1957.

Aesopica, vol. I, ed. B. E. Perry, Urbana, IL 1952.

Epigrammatum Anthologia Palatina cum Planudeis et Appendice nova, vol. I, ed. F. Dübner, Paris 1871.

The Greek Anthology, The Garland of Philip and some contemporary epigrams. Vol. 1. Introduction, Text and Translation, Indexes of Sources and Epigrammatists, ed. by A. S. F. Gow/D. L. Page, London 1968.

Apollonios Rhodios, Argonautica, ed. H. Fränkel, Oxford 1961.

Aristophanis Fabulae, vol. I, ed. N. G. Wilson, Oxford 2007.

Aristoteles, Historia animalium, ed. D. M. Balme, prepared for publication by A. Gotthelf, Cambridge 2011.

Bucolici Graeci, ed. A. S. F. Gow, Oxford 1952.

M. Tullius Cicero, Cato maior. Laelius. De gloria, ed. O. Plasberg/K. Simbeck, München/Leipzig 2005.

L. Iunius Moderatus Columella, Res rustica, ed. R. H. Rodgers, Oxford 2010.

Geoponica, ed. H. Beckh, Leipzig 1895.

Homer, llias, pars I et II, ed. M. L. West, Stuttgart/Leipzig 1998-2000.

M. Annaeus Lucanus, De bello civili, ed. D. R. Shackleton Bailey, Stuttgart 1988.

Palladius Rutilius Taurus Aemilianus, Opus agriculturae. De veterinaria medicina. De institione, ed. R. H. Rodgers, Leipzig 1975.

Pausanias, Graeciae descriptio, vol. I, ed. M. H. Rocha-Pereira, Leipzig 1973.

Platonis Opera, vol. IV, ed. J. Burnet, Oxford 1902.

C. Plinius Secundus, Naturalis historia, vol. II et III, ed. C. Mayhoff, Leipzig 1875 und 1892.

Poetae Melici Graeci (PMG), ed. D. L. Page, Oxford 1962.

[M. Fabius Quintilianus] Declamationes XIX maiores Quintiliano falso ascriptae, ed. L. Håkanson, Stuttgart 1982.

Servii Grammatici qui feruntur in Vergilii Bucolica et Georgica commentarii, rec. G. Thilo, Leipzig 1927.

P. Papinius Statius, Thebais et Achilleis, ed. H. W. Garrod, Oxford 1906.

Suda, pars IV, ed. A. Adler, Leipzig 1935.

M. Terentius Varro, Gespräche über die Landwirtschaft. Herausgegeben und übersetzt von

D. Flach, Darmstadt 2006.

P. Vergili Maronis Opera, rec. R. A. B. Mynors, Oxford 1969.

M. G. Vida, De Bombyce, Rom 1527.

S. Amigues, Théophraste, Recherches sur les plantes, tome III, Paris 1993.

H. Beckby, Anthologia Graeca. Griechisch-Deutsch. 4 Bände, München ${ }^{2} 1965$.

A. R. Benner/F. H. Fobes, The Letters of Alciphron, Aelian and Philostratus, Cambridge, MA 1949.

D. Berrens, Soziale Insekten in der Antike. Ein Beitrag zu Naturkonzepten in der griechisch-römischen Kultur, Göttingen 2018.

D. Berrens, „Der korykische Greis in der Anthologia Graeca”, Hermes (im Druck). 
E. Berti, „Declamazione e poesia“, in: M. Lentano (Hg.), La declamazione latina. Prospettive a confronto sulla retorica di scuola a Roma antica, Neapel 2015, 19-57.

A. Biotti, Virgilio, Georgiche libro IV, Bologna 1994.

E. Burck, „Der korykische Greis in Vergils Georgica (IV 116-148)“, in: F. Jacoby (Hg.), Navicula Chiloniensis, Leiden/Princeton 1956, 156-172.

F. Citti, „Quaedam iura non lege, sed natura. Nature and Natural Law in Roman Declamation”, in: E. Amato/F. Citti/B. Huelsenbeck (Hgg.), Law and Ethics in Greek and Roman Declamation, Berlin/Boston 2015, 95-131.

W. V. Clausen, A Commentary on Virgil Eclogues, Oxford 1994.

J. S. Clay, „The Old Man in the Garden. Georgic 4.116-148“, in: T. M. Falkner/J. de Luce (Hgg.), Old Age in Greek and Roman Literature, New York 1989, 183-194.

R. Coleman, Vergil, Eclogues, Cambridge 1977.

R. Cramer, Vergils Weltsicht. Optimismus und Pessimismus in Vergils Georgica, Berlin/New York 1998.

E. Crane, The Archaeology of Beekeeping, Ithaka, NY 1983.

E. Crane, The World History of Beekeeping and Honey Hunting, New York 1999.

A. Cucchiarelli, Publio Virgilio Marone, Le bucoliche, Rom 2012.

M. Davies/J. Kathirithamby, Greek Insects, New York/Oxford 1986.

K. J. Dover, Aristophanes, Clouds, Oxford 1968.

A. T. Drago, „Api infedeli e fiori avvizziti (Ael. Ep. 5)“, in: O. Vox (Hg.), Lettere, mimesi, retorica, Lecce 2013, 311-325.

M. Erren, P. Vergilius Maro, Georgica. Band 2. Kommentar, Heidelberg 2003.

D. Flach, Marcus Terentius Varro. Gespräche über die Landwirtschaft. Buch III, Darmstadt 2002.

S. Föllinger, „Die antike Biologie zwischen Sachtext und christlicher Predigt: Autoren, Rezipienten und die Frage nach dem literarischen Genus“, in: M. Horster/C. Reitz (Hgg.), Antike Fachschriftsteller, Stuttgart 2003, 72-87.

H. M. Fraser, Beekeeping in Antiquity, London ${ }^{2} 1951$.

M. R. Gale, Virgil on the Nature of Things. The Georgics, Lucretius and the Didactic Tradition, New York ${ }^{2} 2006$.

A. S. F. Gow/D. L. Page, The Greek Anthology, The Garland of Philip and Some Contemporary Epigrams. Vol. 2. Commentary and Indexes, London 1968.

C. Guiraud, Varron, Économie rurale. Livre III, Paris 1997.

K. Gutzwiller, Poetic Garlands. Hellenistic Epigrams in Context, Berkeley 1998.

L. Håkanson, „Die quintilianischen Deklamationen in der neueren Forschung“, ANRW II 32.4, 1986, 2272-2306.

Y. Haskell, „Work or Play? Latin ,Recreational“ Georgic Poetry of the Italian Renaissance“, HumLov 48, 1999, 123-159.

Y. Haskell, Loyola's bees. Ideology and Industry in Jesuit Latin Didactic Poetry, Oxford 2003.

D. B. Hollander, Farmers and Agriculture in the Roman Economy, London/New York 2019.

N. Holzberg, Die antike Fabel. Eine Einführung, Darmstadt ${ }^{3} 2012$.

R. L. Hunter, Theocritus, a Selection. Idylls 1, 3, 4, 6, 7, 10, 11 and 13, Cambridge 1999.

J. E. Jones, „Hives and Honey of Hymettus. Beekeeping in Ancient Greece“, Archaeology 29.2, 1976, 80-91.

J. F. Kindstrand, „Claudius Aelianus und sein Werk“, ANRW II 34.4, 1998, 2954-2996.

J. Klek, „Die Bienenkunde des Altertums IV. Die Spätzeit“, Archiv für Bienenkunde 7, 1926, 47-57.

J. Klek/L. Armbruster, „Die Bienenkunde des Aristoteles und seiner Zeit. Übersetzung, Einleitung und geschichtlich-sprachliche Anmerkungen“, Archiv für Bienenkunde 1, 1919, 185-240. 
J. Klek/L. Armbruster, „Varro und Vergil“, Archiv für Bienenkunde 2, 1920, 243-291.

J. Klek/L. Armbruster, „Columella und Plinius“, Archiv für Bienenkunde 3, 1921, 286-318.

F. Klingner, Virgil. Bucolica, Georgica, Aeneis, Zürich/Stuttgart 1967.

R. König/G. Winkler, Plinius, Naturkunde. Bücher XXI und XXII. Medizin und Pharmakologie: Heilmittel aus dem Pflanzenreich, München ${ }^{2} 1999$.

G. Krapinger, [Quintilian], Die Bienen des armen Mannes. (Größere Deklamationen, 13), Cassino 2005.

G. Krapinger, „Die Bienen des armen Mannes in Antike und Mittelalter“, in: M. Grünbart (Hg.), Theatron, Berlin 2007, 189-201.

W. Kullmann, „Zoologische Sammelwerke in der Antike“, in: W. Kullmann/J. Althoff/M. Asper (Hgg.), Gattungen wissenschaftlicher Literatur in der Antike, Tübingen 1998, 121-139.

A. La Penna, „Senex Corycius“, in: Instituo Universitario Orientale (Hg.), Atti del Convegno Virgiliano sul bimillenario delle Georgiche, Napoli 1977, 37-66.

J. G. Lennox, „The Disappearance of Aristotle’s Biology. A Hellenistic Mystery“, in: T. D. Barnes (Hg.), The Sciences in Greco-Roman Society, Edmonton 1994, 7-24.

P. L. M. Leone, „Le lettere rustiche di Claudio Eliano. Introduzione, traduzione e note“, AFLL 7 (1975-76), 1977, 55-74.

D. Mantovani, „I giuristi, il retore e le api. lus conteroversum e natura nella Declamatio maior XIII“, in: D. Mantovani/A. Schiavone (Hgg.), Testi e problemi del giusnaturalismo romano, Pavia 2007, 323-385.

C. de Meo, Giovanni Pascoli, Senex Corycius (Cilix). Introduzione, testo e traduzione commento, Bologna 1974.

R. Merkelbach, „Zu-“, Glotta 45, 1967, 39-40.

R. Monreal, „Vergils Vermächtnis. Die Gartenpraeteritio in den ,Georgica“ (4,116-148) und Typen ihrer Rezeption im neulateinischen Lehrgedicht“, HumLov 54, 2005, 1-47.

J. Moore-Blunt, „Two Epigrams on Animals“, AJPh 98, 1977, 351.

R. A. B. Mynors, Virgil, Georgics, Oxford 1990.

H-G. Nesselrath, Platon, Kritias, Göttingen 2006.

M. Nøjgaard, La fable antique. Tome Premier. La fable grecque avan Phèdre, Kopenhagen 1964.

T. Olbertz, „,Illum admirantur et omnes'. Apis in der klassischen römischen Literatur“, in: D. Engels/C. Nicolaye (Hgg.), Ille operum custos. Kulturgeschichtliche Beiträge zur antiken Bienensymbolik und ihrer Rezeption., Hildesheim 2008, 95-113.

F. Olck, „Bienenzucht“, RE 3, 1897, 450-457.

F. Pelliccio, Gli epigrammi di Apollonide. Introduzione, Tradizione e Commento, Diss. Neapel 2013.

C. G. Perkell, „The Corycian Gardener of Vergil's Fourth Georgic“, TAPhA 111, 1981, 167-177.

J. G. F. Powell, Cicero, Cato maior. De senectute. Edited with Introduction and Commentary, Cambridge 1988.

M. C. J. Putnam, Virgil's Pastoral Art. Studies in the Eclogues, Princeton, NJ 1970.

C. Reitz, Zur Gleichnistechnik des Apollonios von Rhodos, Bern/Frankfurt a. M. 1996.

C. Ritter, Die quintilianischen Declamationen. Untersuchung über Art und Herkunft derselben, Freiburg/Tübingen 1881.

E. de Saint-Denis, „Une source de Virgile dans les Géorgiques“, REL 16, 1938, 297-317.

A. Salvatore, „Georgiche di Virgilio e De re rustica di Varrone“, in: Instituo Universitario Orientale (Hg.), Atti del Convegno Virgiliano sul bimillenario delle Georgiche, Napoli 1977, 67-111.

S. D. Smith, Man and Animal in Severan Rome. The Literary Imagination of Claudius Aelianus, Cambridge 2014. 
A. H. Sommerstein, Aristophanes, Clouds, Warminster 1982.

S. A. Stephens, Callimachus, The Hymns. Edited with Introduction, Translation, and Commentary, Oxford/New York 2015.

J. Sünskes Thompson, „Bienenzucht“, in: Der Neue Pauly 2, 1997, 650-652.

R. Tabacco, „Povertà e ricchezza. L'unità tematica della declamazione XIII dello Pseudo-Quintiliano“, MCSN 2, 1978a, 37-70.

R. Tabacco, „L'utilizzazione dei topoi nella declamazione XIII dello Pseudo-Quintiliano“, AAT112, 1978 b, 197-224.

R. Tabacco, „Ps.-Quint. XIII 15. Un problema di interpretazione“, BStudLat 9, 1979, 279-280.

P. Thibodeau, „The Old Man and His Garden (Verg. Georg. 4, 116-148)“, MD 47, 2001, 175-195.

R. F. Thomas, Virgil's Georgics. Volume 2. Books III-IV, Cambridge 1988.

R. F. Thomas, „The Old Man Revisited. Memory, Reference and Genre in Virg., Georg. 4, 116-48“, MD 28, 1992, 35-70.

B. Tilly, Varro the farmer. A Selection from the Res rusticae, London 1973.

P. Waltz, Anthologie grecque. Tome III (Livre VI), Paris 1931.

P. Waltz/G. Soury, Anthologie grecque. Tome VIII (Livre IX, 359-827), Paris 1974.

A. Wilhelm, „Anthologia Palatina VI 238“, AC 4, 1935, 449-455.

L. P. Wilkinson, The Georgics of Virgil. A Critical Survey, Cambridge 1969. 